\title{
Product group confinement in SUSY gauge theories
}

\author{
Benjamin Lillard \\ Department of Physics $\&$ Astronomy, University of California, \\ Irvine Fredrick Reines Hall, Irvine, CA, 92697 U.S.A. \\ E-mail: blillard@uci.edu
}

ABSTRACT: We propose a new set of s-confining theories with product gauge groups and no tree-level superpotential, based on a model with one antisymmetric matter field and four flavors of quarks. For each product group we find a set of gauge-invariant operators which satisfy the 't Hooft anomaly matching conditions, and we identify the dynamically generated superpotential which reproduces the classical constraints between operators. Several of these product gauge theories confine without breaking chiral symmetry, even in cases where the classical moduli space is quantum-modified. These results may be useful for composite model building, particularly in cases where small operators of the form $(Q \bar{Q})$ are absent, or for theories with multiple natural energy scales, and may provide new ways to break supersymmetry dynamically.

Keywords: Confinement, Supersymmetric Gauge Theory, Supersymmetry and Duality

ARXIV EPRINT: 1704.06282 


\section{Contents}

1 Introduction 1

1.1 Review: Seiberg dualities 2

1.2 Review: s-confinement 3

$1.3 \mathrm{SU}(N)$ with antisymmetric tensor 5

2 Product group extension for an s-confining theory $\quad 8$

2.1 Infrared operators $\quad 9$

$\begin{array}{ll}2.2 & \text { Dynamically generated superpotential } \\ & 14\end{array}$

$\begin{array}{lll}2.3 & \text { Additional tests } & 17\end{array}$

3 Other s-confining theories $\quad 20$

3.1 Special case: $\mathrm{SU}(4) \quad 21$

$\begin{array}{lll}3.2 & \mathrm{Sp}(2 m) \text { with }(2 m+4) \text { quarks } & 27\end{array}$

$\begin{array}{lll}3.3 & \text { SUSY QCD } & 28\end{array}$

3.4 Other models 30

4 Conclusion $\quad 31$

A Derivation of classical constraints

A.1 D-flat directions 33

A.2 Special cases 34

\section{Introduction}

Experimental evidence so far suggests that the Standard Model gauge group $G_{\mathrm{SM}}=$ $\mathrm{SU}(3)_{c} \times \mathrm{SU}(2)_{L} \times \mathrm{U}(1)_{Y}$ well describes the universe. Attempts to expand the gauge sector beyond $G_{\mathrm{SM}}$ must therefore explain why the additional interactions have not yet presented any evidence for their existence.

There are several well-motivated ways to achieve this. The new gauge bosons and matter fields might form a "dark sector" and interact weakly (or not at all) with the particles described by the Standard Model. It is also possible for an extended gauge symmetry to be spontaneously broken to $G_{\mathrm{SM}}$ at some high-energy scale which we have not yet probed. In this paper we consider an alternative in which the new dynamics are so strongly coupled that particles charged under the new interactions confine to form neutral bound states, with binding energies at the $\mathrm{TeV}$ scale or larger.

We focus on a particular class of $\mathcal{N}=1$ supersymmetric (SUSY) gauge theories with product gauge groups of the form $\mathrm{SU}(N)_{1} \times \mathrm{SU}(N)_{2} \times \ldots \times \mathrm{SU}(N)_{k}$. Our model includes one antisymmetric tensor $A_{\alpha \beta}$ and four quark fields $Q_{\alpha}^{i}$ charged under $\mathrm{SU}(N)_{1}$, and a series 


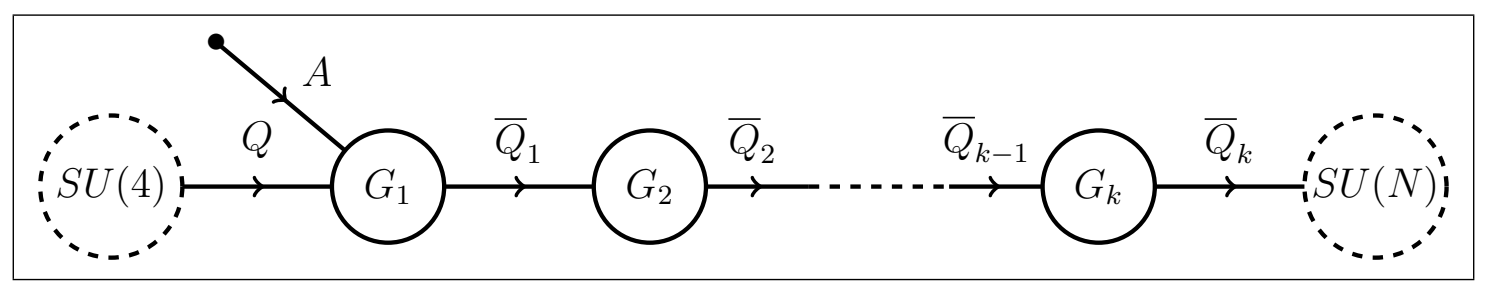

Table 1. The matter content of the proposed s-confining theory is shown as a moose diagram. Each $G_{i}$ represents a gauged $\mathrm{SU}(N)$ group, while the dashed circles represent the $\mathrm{SU}(4)_{L} \times \mathrm{SU}(N)_{R}$ family symmetry.

of bifundamental fields $\left(\bar{Q}_{i}\right)_{\beta}^{\alpha}$ charged under adjacent gauge groups $\mathrm{SU}(N)_{i} \times \mathrm{SU}(N)_{i+1}$ as shown in table 1 . This theory is an extension of a model, $\mathrm{SU}(N):(\square+4 \square+N \bar{\square})$, which has been shown to confine [1-3].

We propose in the language of [4] that this $\mathrm{SU}(N)^{k}$ model is "s-confining": that is, the theory confines smoothly in the infrared without breaking chiral symmetry, and it generates a non-vanishing superpotential that describes the interactions between the gauge invariant composite fields. Although the $\mathcal{N}=1$ s-confining theories with a simple gauge group are fully classified [5], very few examples of s-confinement in product gauge groups are known $[6,7]$.

Our SU $(N)^{k}$ product group model has two distinctive features which may be useful for model-building. First, there are no small gauge-singlet operators: the number of fields contained in every gauge invariant operator depends on $k$ or $N$. Second, the various $\operatorname{SU}(N)_{i}$ subgroups generally confine at different scales $\Lambda_{i}$, with hierarchies based on the coupling constants $g_{i}$.

Product groups of this form appear in studies of five-dimensional gauge theories [811]. The model shown in table 1 can be interpreted as a $k$-site deconstruction of a $5 \mathrm{~d}$ SUSY $\mathrm{SU}(N)$ gauge theory with a $\mathbb{Z}_{2}$ orbifold. In the $5 \mathrm{~d}$ theory the chiral fields $\{A, Q\}$ and $\bar{Q}_{k}$ exist on opposing $4 \mathrm{~d}$ branes, while the bifundamental $\bar{Q}_{i}$ superfields correspond to a single bulk $\bar{Q}$ field. A natural hierarchy between the $\Lambda_{i}$ arises if the extra dimension is warped: for example, the model with $\Lambda_{1}>\ldots>\Lambda_{k}$ has $A$ and $Q$ on the ultraviolet brane and $\bar{Q}_{k}$ on the infrared brane.

In sections 1.1 and 1.2 we review the basic aspects of confining SUSY gauge theories. In section 1.3 we discuss more specific properties of the $A+4 Q+N \bar{Q}$ model with an $\operatorname{SU}(N)$ gauge group, including the coefficients in its dynamically generated superpotential. These coefficients do not appear in the literature, so we include our derivation in appendix A. Section 2 contains a detailed discussion of the $\mathrm{SU}(N)^{k}$ product gauge group models and our primary results. In section 3 we suggest other product group models which may be s-confining, as well as several counter-examples.

\subsection{Review: Seiberg dualities}

It is generally difficult to analyze the infrared behavior of strongly coupled theories, due to the failure of perturbation theory in this limit. Seiberg, Intriligator and others have made this problem more tractable by exploiting some of the remarkable properties of 
supersymmetry, allowing some infrared properties of SUSY gauge theories to be calculated exactly $[12,13]$. Seiberg's infrared dualities between different phases of gauge theories were central to these developments. We summarize some of the results in this section; a more detailed review is given in [14].

Seiberg found that in $\mathrm{SU}(N)$ gauge groups with $F$ flavors of quarks and antiquarks, also known as SUSY QCD, the infrared behavior of the $F=N$ and $F=N+1$ cases can be completely described by a set of gauge invariant operators, $M=Q \bar{Q}, B=Q^{N}$, and $\bar{B}=\bar{Q}^{N}$. This dual theory has no gauge interactions, so the $F=N$ and $F=N+1$ theories are said to confine: every test charge can be "screened" by creating quark-antiquark pairs from the vacuum, and a gauge-invariant Wilson loop obeys a perimeter law.

Classically, the gauge invariant operators obey particular constraints, following from the Bose symmetry of the superfields and the definitions of $M, B$, and $\bar{B}$. For $F=N+1$,

$$
\begin{aligned}
B_{i} M_{j}^{i} & =0 \\
M_{j}^{i} \bar{B}^{j} & =0 \\
\left(M_{j}^{i}\right)^{-1} \operatorname{det} M & =B_{i} \bar{B}^{j},
\end{aligned}
$$

while for $F=N$

$$
\operatorname{det} M-B \bar{B}=0,
$$

where the indices $i$ and $j$ refer to the family $\mathrm{SU}(F)$ symmetries of the $Q$ and $\bar{Q}$. It has been shown [15-17] that eq. (1.2) is modified quantum mechanically:

$$
\operatorname{det} M-B \bar{B}=\Lambda^{b},
$$

where $\Lambda^{b}$ is the holomorphic scale

$$
\Lambda^{b}=\mu^{b} \exp \left\{-8 \pi^{2} / g^{2}+i \theta_{\mathrm{YM}}\right\} .
$$

Here $\theta_{\mathrm{YM}}$ is the $C P$-violating $\theta$-term of the $\mathrm{SU}(N)$ gauge group, $g$ is the gauge coupling, and $b=3 N-F=2 N$ is derived from the $\beta$ function for the gauge coupling. The quantum-modified constraint eq. (1.3) can be enforced by a superpotential

$$
W=\lambda\left(\operatorname{det} M-B \bar{B}-\Lambda^{2 N}\right)
$$

if we introduce a Lagrange multiplier superfield $\lambda$. At the origin of the classical moduli space, $M=B=\bar{B}=0$, the UV family symmetry $\mathrm{SU}(F)_{L} \times \mathrm{SU}(F)_{R} \times \mathrm{U}(1)_{B}$ is conserved. However, this point is not on the quantum-deformed moduli space given by eq. (1.3), so the chiral symmetry is broken in the vacuum.

\subsection{Review: s-confinement}

In the $F=N+1$ case, the classical constraint equations are not modified. Instead, they are enforced by a dynamically generated superpotential [18]:

$$
W_{d}=\frac{1}{\Lambda^{2 N-1}}[B M \bar{B}-\operatorname{det} M]
$$


which has $\langle M\rangle=\langle B\rangle=\langle\bar{B}\rangle=0$ as a solution to the equations of motion. This vacuum corresponds to confinement without chiral symmetry breaking, which we refer to as sconfinement. More precisely, a theory is s-confining if [4]:

- All infrared degrees of freedom are gauge invariant composite fields;

- The infrared physics is described by a smooth effective theory, which is valid everywhere on the moduli space (including the origin);

- There is a dynamically generated superpotential.

For the effective theory to be smooth, there should be no gauge invariant order parameter that can distinguish the Higgs and confined phases of the theory. The infrared degrees of freedom must also satisfy the anomaly matching conditions.

Generally, the dynamically generated superpotential is determined up to an overall factor based on symmetry arguments, and by matching its equations of motion to the classical constraints. Its dependence on the holomorphic scale $\Lambda^{b}$ can be found either on dimensional grounds, or by requiring that $W_{d}$ is neutral under the anomalous $\mathrm{U}(1)$ symmetry.

The requirement that a superpotential is dynamically generated adds a powerful constraint on the matter content of any s-confining theory. An $\mathcal{N}=1$ susy theory with $f$ massless matter superfields has a classical family symmetry of rank $f+1$ including the $R$ symmetry, but the $G^{2} \mathrm{U}(1)$ anomaly removes one linear combination of the $\mathrm{U}(1)$ family symmetries. This allows us to define a $\mathrm{U}(1)_{R}$ symmetry such that exactly one of the matter superfields $\phi_{i}$ has $R$ charge, $q_{i}$, with all other fields neutral. Using the normalization in which the gauginos have $R$ charge +1 , cancellation of the $G^{2} \mathrm{U}(1)_{R}$ anomaly requires that

$$
q_{i}=\frac{1}{\mu_{i}}\left[\sum_{j} \mu_{j}-\mu_{G}\right],
$$

where $\mu_{j}$ and $\mu_{G}$ are the Dynkin indices of the matter fields $\phi_{j}$ and the gluinos, respectively, with the normalization $\mu(\square)=1$. For the dynamically generated superpotential to have $R$ charge +2 under any of the possible anomaly-free $R$ symmetries, it must have the form

$$
W \sim \prod_{i}\left[\phi_{i}^{2 / q_{i}}\right]=\prod_{i}\left(\phi_{i}^{\mu_{i}}\right)^{2 /\left[\sum_{j} \mu_{j}-\mu_{G}\right]} .
$$

The matter content must therefore satisfy the index constraint of Csaki et al. [4]:

$$
\sum_{j} \mu_{j}-\mu_{G}=2 .
$$

In [5] this index constraint is used to find all $\mathcal{N}=1 \mathrm{~s}$-confining theories with one gauge group and no tree-level superpotential. Both $F=N+1$ SUSY QCD and the $A+4 Q+N \bar{Q}$ model are included.

In theories with a product gauge group this constraint is relaxed: the number of fields exceeds the rank of the family symmetry, and it is no longer possible to identify a unique $R$ symmetry for each field. 


\section{3 $\mathrm{SU}(N)$ with antisymmetric tensor}

Properties of the $\boxminus+F \square+(N+F-4) \square$ model have been studied by several authors [1, $2,19,20]$. In the $F=2$ case there is a superpotential generated by a one-instanton effect; for $F=3$ the theory confines, with a quantum-deformed moduli space that induces dynamical symmetry breaking; and for $F=4$, the theory is s-confining. The quantum modified constraints have been derived in [19] for $F=3$, but the classical constraints for the $A+4 Q+N \bar{Q}$ model do not appear in the literature. We derive the relative coefficients of the dynamically generated superpotential in appendix A, and quote the results in this section.

Infrared operators. In the $A+4 Q+N \bar{Q}$ model, the set of gauge invariant operators changes based on whether $N$ is even or odd. This is due to the $\boxminus$ representation: if $N=2 m$ is even, then the gauge invariants include the antisymmetrized products $\left(A^{m}\right)$, $\left(A^{m-1} Q^{2}\right)$, and $\left(A^{m-2} Q^{4}\right)$, while for odd $N=2 m+1$ the gauge invariants include $\left(A^{m} Q\right)$ and $\left(A^{m-1} Q^{3}\right)$.

Below, we define the simplest gauge invariant operators for the $N=2 m$ and $N=2 m+1$ models. Both cases include the operators $(Q \bar{Q}),\left(A \bar{Q}^{2}\right)$, and $\left(\bar{Q}^{N}\right)$ :

$$
\begin{aligned}
J_{j}^{i} & =Q_{\alpha}^{i} \bar{Q}_{j}^{\alpha}, \\
K_{j_{1} j_{2}} & =A_{\alpha \beta} \bar{Q}_{j_{1}}^{\alpha} \bar{Q}_{j_{2}}^{\beta}, \\
Z & =\operatorname{det} \bar{Q}=\frac{\epsilon_{\alpha_{1} \ldots \alpha_{N}} \epsilon^{j_{1} \ldots j_{N}}}{N !}\left(\bar{Q}_{j_{1}}^{\alpha_{1}} \bar{Q}_{j_{2}}^{\alpha_{2}} \ldots \bar{Q}_{j_{N}}^{\alpha_{N}}\right) .
\end{aligned}
$$

For even $N \geq 4$, we also add the gauge invariants

$$
\begin{aligned}
U & =\operatorname{Pf} A=\frac{\epsilon^{a_{1} a_{2} \ldots a_{N}}}{2^{m} m !}\left(A_{a_{1} a_{2}} A_{a_{3} a_{4}} \ldots A_{a_{N-1} a_{N}}\right) \\
V_{i_{1} i_{2}} & =\frac{\epsilon^{a_{1} a_{2} \ldots a_{N}}}{2^{m-1}(m-1) ! 2 !}\left(A_{a_{1} a_{2}} A_{a_{3} a_{4}} \ldots A_{a_{N-3} a_{N-2}}\right) Q_{a_{N-1}}^{i_{1}} Q_{a_{N}}^{i_{2}}, \\
\mathcal{W} & =\frac{\epsilon^{a_{1} a_{2} \ldots a_{N}}}{2^{m-2}(m-2) !} \frac{\epsilon_{j_{1} j_{2} j_{3} j_{4}}}{4 !}\left(A_{a_{1} a_{2}} A_{a_{3} a_{4}} \ldots A_{a_{N-5} a_{N-4}}\right) Q_{a_{N-3}}^{j_{1}} Q_{a_{N-2}}^{j_{2}} Q_{a_{N-1}}^{j_{3}} Q_{a_{N}}^{j_{4}},
\end{aligned}
$$

whereas for odd $N \geq 5$ we include

$$
\begin{aligned}
X^{j} & =\frac{\epsilon^{a_{1} a_{2} \ldots a_{N}}}{2^{m} m !}\left(A_{a_{1} a_{2}} A_{a_{3} a_{4}} \ldots A_{a_{N-2} a_{N-1}}\right) Q_{a_{N}}^{j}, \\
Y_{j} & =\frac{\epsilon^{a_{1} a_{2} \ldots a_{N}}}{2^{m-1}(m-1) !} \frac{\epsilon_{j j_{2} j_{3} j_{4}}}{3 !}\left(A_{a_{1} a_{2}} A_{a_{3} a_{4}} \ldots A_{a_{N-4} a_{N-3}}\right) Q_{a_{N-2}}^{j_{2}} Q_{a_{N-1}}^{j_{3}} Q_{a_{N}}^{j_{4}} .
\end{aligned}
$$

The numeric coefficients absorb the combinatoric factors from the $\epsilon$ tensors, with the convention $\epsilon_{123 \ldots N}=+1$. In general, we reserve the indices $a, b, \alpha, \beta$ for gauge groups, and use the indices $i, j$ to refer to family symmetries. Superscripts and subscripts are chosen for visual clarity, and do not signify any particular group representation.

It is useful to classify the $\{U, V, \mathcal{W}, X, Y, Z\}$ fields as "baryons" and the $J$ and $K$ fields as "mesons", to separate the operators which scale with $N$ from those which are 


\begin{tabular}{|c|c|cc|ccc|c|}
\hline & $G$ & $\mathrm{SU}(4)_{L}$ & $\mathrm{SU}(N)_{R}$ & $U_{A}$ & $U_{B}$ & $U_{R}$ & $U_{1}$ \\
\hline$A$ & $\square$ & & & -4 & -1 & 0 & 0 \\
$Q$ & $\square$ & $\square$ & & $N-2$ & $-1 / 2$ & $1 / 2$ & 0 \\
$\bar{Q}$ & $\square$ & & $\square$ & 0 & 1 & 0 & 1 \\
\hline$\Lambda^{b}$ & & & & 0 & 0 & 0 & $N$ \\
\hline$J$ & & $\square$ & $\square$ & $N-2$ & $1 / 2$ & $1 / 2$ & 1 \\
$K$ & & & $\square$ & -4 & 1 & 0 & 2 \\
\hline$Z$ & & & & 0 & $N$ & 0 & $N$ \\
\hline$U$ & & & & $-2 N$ & $-N / 2$ & 0 & 0 \\
$V$ & & $\square$ & & 0 & $-N / 2$ & 1 & 0 \\
$\mathcal{W}$ & & & & $2 N$ & $-N / 2$ & 2 & 0 \\
\hline$X$ & & $\square$ & & $-N$ & $-N / 2$ & $1 / 2$ & 0 \\
$Y$ & & $\square$ & & $N$ & $-N / 2$ & $3 / 2$ & 0 \\
\hline
\end{tabular}

Table 2. The transformation properties of the UV and IR fields under the family $\mathrm{SU}(4)_{L} \times$ $\mathrm{SU}(N)_{R} \times \mathrm{U}(1)_{A} \times \mathrm{U}(1)_{B} \times \mathrm{U}(1)_{R}$ symmetry for the $F=4$ model are shown, along with the charges under the spurious $\mathrm{U}(1)_{1}$. The operators $J, K$, and $Z$ are defined whether $N$ is even or odd; the fields $U, V$ and $\mathcal{W}$ are specific to the even $N$ case, while the fields $X$ and $Y$ correspond to the odd $N$ case. The $\mathrm{U}(1)_{R}$ charges listed refer to the scalar component of each superfield.

independent of $N$. The transformation properties of these operators under the family symmetries are shown in table 2 . There is a continuous family of equivalent $\mathrm{U}(1)_{A} \times \mathrm{U}(1)_{B} \times$ $\mathrm{U}(1)_{R}$ charge assignments, but the choice shown in table 2 is particularly convenient.

For $N=4$, the theory contains four flavors of $Q+\bar{Q}$. This value of $N$ is unique in that both $m_{A} \operatorname{Pf} A$ and $m_{j}^{i} Q_{i}^{\alpha} \bar{Q}_{\alpha}^{j}$ are gauge-invariant mass terms: if these masses are large compared to $\Lambda$, then every field can be integrated out above the confinement scale. This special case is discussed in section 3.1. For $N=3$ the $\boxminus$ and $\square$ representations are equivalent, and the $A+4 Q+3 \bar{Q}$ model reduces to SUSY QCD with $F=4$.

As discussed in section 1.1, the form of the dynamically generated superpotential is determined by the representations of the matter fields. For the $A+4 Q+N \bar{Q}$ model,

$$
W_{d} \sim \sum \frac{A^{N-2} Q^{4} \bar{Q}^{N}}{\Lambda^{b}}
$$

The sum includes all possible gauge-invariant contractions of the group indices, with some relative coefficients:

$$
\begin{aligned}
W_{\text {odd N }} & \sim \frac{1}{\Lambda^{b}}\left[X Y Z+X K^{m-1} J^{3}+Y K^{m} J\right] \\
W_{\text {even N }} & \sim \frac{1}{\Lambda^{b}}\left[U \mathcal{W} Z+V^{2} Z+U K^{m-2} J^{4}+V K^{m-1} J^{2}+\mathcal{W} K^{m}\right] .
\end{aligned}
$$


Both $\mathcal{F}_{\text {odd }}=\{J, K, X, Y, Z\}$ and $\mathcal{F}_{\text {even }}=\{J, K, U, V, \mathcal{W}, Z\}$ satisfy the t' Hooft anomaly matching conditions for the mixed $\mathrm{SU}(4)^{2} \mathrm{U}(1)$ and $\mathrm{SU}(N)^{2} \mathrm{U}(1)$ anomalies, the various $\mathrm{U}(1)^{3}$ anomalies, and the mixed $\mathrm{U}(1)$ gravitational anomalies, for all $\mathrm{U}(1)$ symmetries listed in table 2 except for $\mathrm{U}(1)_{1}$. The $G_{1}^{2} \mathrm{U}(1)_{1}$ anomaly breaks $\mathrm{U}(1)_{1}$ explicitly at the scale $\Lambda_{1}$, so it is not a symmetry of the infrared theory.

Dynamically generated superpotential. The number of infrared operators, $\operatorname{dim} \mathcal{F}$, is larger than the dimension of the moduli space, $\operatorname{dim} M_{0}=N(N-1) / 2+4 N+1$. For $N=2 m+1$,

$$
\operatorname{dim}\{J, K, X, Y, Z\}=\left(4 N+\frac{N(N-1)}{2}+4+4+1\right)
$$

and for $N=2 m$,

$$
\operatorname{dim}\{J, K, U, V, \mathcal{W}, Z\}=\left(4 N+\frac{N(N-1)}{2}+1+\frac{4(3)}{2}+1+1\right)
$$

implying for both cases that the number of constraints is

$$
N_{\text {con }}=\operatorname{dim} \mathcal{F}-\operatorname{dim} M_{0}=8
$$

For odd $N$, the eight constraints are

$$
\begin{aligned}
X^{i} Z & =\frac{\epsilon^{j_{1} j_{2} \ldots j_{N}}}{2^{m} m !}\left(K_{j_{1} j_{2}} K_{j_{3} j_{4}} \ldots K_{j_{N-2} j_{N-1}}\right) J_{j_{N}}^{i} \\
Y_{i} Z & =\frac{\epsilon^{j_{1} j_{2} \ldots j_{N}} \epsilon_{i i_{2} i_{3} i_{4}}}{2^{m-1}(m-1) ! 3 !}\left(K_{j_{1} j_{2}} K_{j_{3} j_{4}} \ldots K_{j_{N-4} j_{N-3}}\right) J_{j_{N-2}}^{i_{2}} J_{j_{N-1}}^{i_{3}} J_{j_{N}}^{i_{4}}
\end{aligned}
$$

while for even $N$

$$
\begin{aligned}
U Z & =\frac{\epsilon_{j_{1} \ldots j_{N}}}{2^{m} m !} K_{j_{1} j_{2}} K_{j_{3} j_{4}} \ldots K_{j_{N-1} j_{N}}=\operatorname{Pf} K \\
V_{i_{1} i_{2}} Z & =\frac{\epsilon_{j_{1} \ldots j_{N}}}{2^{m-1}(m-1) !} \frac{\epsilon_{i_{1} i_{2} i_{3} i_{4}}}{2 !} K_{j_{1} j_{2}} K_{j_{3} j_{4}} \ldots K_{j_{N-3} j_{N-2}} J_{j_{N-1}}^{i_{3}} J_{j_{N}}^{i_{4}}, \\
\mathcal{W} Z & =\frac{\epsilon_{j_{1} \ldots j_{N}}}{2^{m-2}(m-2) !} \frac{\epsilon_{i_{1} i_{2} i_{3} i_{4}}}{4 !} K_{j_{1} j_{2}} K_{j_{3} j_{4}} \ldots K_{j_{N-5} j_{N-4}} J_{j_{N-3}}^{i_{1}} J_{j_{N-2}}^{i_{2}} J_{j_{N-1}}^{i_{3}} J_{j_{N}}^{i_{4}} .
\end{aligned}
$$

The index $i=1 \ldots 4$ refers to the $\mathrm{SU}(4)$ family symmetry.

By taking partial derivatives of eq. (1.19) and eq. (1.20) and matching the equations of motion to the classical constraints, one can determine the relative coefficient of each term in the dynamically generated superpotential. The results appear below:

$$
\begin{aligned}
W_{\text {odd }}= & \frac{\alpha}{\Lambda^{b}}\left\{X^{i} Y_{i} Z-\frac{\epsilon^{j_{1} \ldots j_{N}} \epsilon_{i_{1} \ldots i_{4}}}{2^{m-1}(m-1) ! 3 !} X^{i_{1}}\left(K_{j_{1} j_{2}} \ldots K_{j_{N-4} j_{N-3}}\right) J_{j_{N-2}}^{i_{2}} J_{j_{N-1}}^{i_{3}} J_{j_{N}}^{i_{4}}\right. \\
& \left.-\frac{\epsilon^{j_{1} \ldots j_{N}}}{2^{m} m !} Y_{i}\left(K_{j_{1} j_{2}} \ldots K_{j_{N-2} j_{N-1}}\right) J_{j_{N}}^{i}\right\}
\end{aligned}
$$




$$
\begin{aligned}
W_{\text {even }}= & \frac{\alpha}{\Lambda^{b}}\left\{U \mathcal{W} Z-\frac{\epsilon_{i_{1} \ldots i_{4}}}{2^{2} 2 !} V^{i_{1} i_{2}} V^{i_{3} i_{4}} Z-\mathcal{W} \operatorname{Pf} K\right. \\
& -\frac{\epsilon_{j_{1} \ldots j_{N}}}{2^{m-2}(m-2) !} \frac{\epsilon_{i_{1} i_{2} i_{3} i_{4}}}{4 !} U\left(K_{j_{1} j_{2}} \ldots K_{j_{N-5} j_{N-4}}\right)\left(J_{j_{N-3}}^{i_{1}} \ldots J_{j_{N}}^{i_{4}}\right) \\
& \left.+\frac{\epsilon_{j_{1} \ldots j_{N}} \epsilon_{i_{1} i_{2} i_{3} i_{4}}}{4 \cdot 2^{m-1}(m-1) !} V^{i_{1} i_{2}}\left(K_{j_{1} j_{2}} \ldots K_{j_{N-3} j_{N-2}}\right) J_{j_{N-1}}^{i_{3}} J_{j_{N}}^{i_{4}}\right\} .
\end{aligned}
$$

As in SUSY QCD, the overall factor $\alpha$ cannot be determined by symmetry arguments. In principle, it is possible to add heavy quark masses and integrate out two flavors of $(Q \bar{Q})$ so as to match the $F=2$ model, whose superpotential can be calculated from a one-instanton calculation analogous to $F=N-1$ SUSY QCD. In our present study we do not perform this calculation.

It is useful, however, to consider the phases of $\alpha$ and $\Lambda^{b}$. As defined in eq. (1.4), the phase of $\Lambda^{b}$ is determined by the $C P$-violating $\theta_{\mathrm{YM}}$ parameter. The phase of $\alpha$ is also unknown: however, because $W_{d}$ is charged under an unbroken $\mathrm{U}(1)_{R}$ symmetry, it can be rotated by a phase without affecting the Lagrangian $\mathcal{L} \sim \int d \theta^{2} W$, so as to make $\alpha$ real.

\section{Product group extension for an s-confining theory}

Our interest in the product group model of table 1 is motivated by an observation from the $G_{1} \times G_{2}$ case, in which the family symmetry $G_{2}=\mathrm{SU}(N)_{R}$ of the $\bar{Q}$ is weakly gauged. In the confined phase of $G_{1}$, there are three types of operators charged under $G_{2}$ : one antisymmetric $K=\emptyset$, four quarks $J=\square$, and $N$ antiquarks $\bar{Q}_{2}=\bar{\square}$. Remarkably, this is identical to the original s-confining model.

The model described in section 1.3 can be extended indefinitely by adding more gauge groups $G_{i}$ and bifundamental matter $\bar{Q}_{i}$. As long as $\Lambda_{1}>\Lambda_{2} \ldots>\Lambda_{i}>\Lambda_{i+1}$, confinement under $G_{i}$ always produces mesons charged as $\boxminus+4 \square$ under $G_{i+1}$. This is the model shown in table 1 , where the gauge group is $G_{1} \times \ldots \times G_{k}$. In this section we devote our attention to the question: is this $\mathrm{SU}(N)^{k}$ theory s-confining, or is s-confinement disrupted by the product group?

There are two obvious ways in which the $K+4 J+N \bar{Q}_{2}$ " $\mathrm{k}=2$ " model differs from the original (" $\mathrm{k}=1$ ") s-confining theory. First, in the $k=1$ model there is no tree-level superpotential, but in the $k=2$ case there is a superpotential from $G_{1}$ confinement that may alter how $\{K, J, P\}$ confine under $G_{2}$. Luckily, inspection of the classical constraints shows that $K, J$, and $\bar{Q}_{2}$ may be varied freely, as long as the baryon products $\{U Z, V Z, \mathcal{W} Z\}$ or $\{X Z, Y Z\}$ vary in accordance with eqs. (1.24) and (1.25). The second main difference is that under $G_{2}$, the classical moduli space is modified quantum mechanically. For the $k \geq 2$ theory to be s-confining, we must determine whether or not the origin remains on the moduli space.

Of the existing literature regarding SUSY product groups, the work of Chang and Georgi [11] on $\mathrm{SU}(N)^{k}$ extensions to $F=N$ SUSY QCD is particularly useful to our present study. Our method also has some similarities to deconfinement [1,21], particularly in section 3 when we consider $\operatorname{Sp}(2 N)$ groups. 


\begin{tabular}{|c|ccccc|cc|ccc|ccccc|}
\hline & $G_{1}$ & $G_{2}$ & $G_{3}$ & $\ldots$ & $G_{k}$ & $\mathrm{SU}(4)$ & $\mathrm{SU}(N)$ & $U_{A}$ & $U_{B}$ & $U_{R}$ & $U_{1}$ & $U_{2}$ & $U_{3}$ & $\ldots$ & $U_{k}$ \\
\hline$Q$ & $\square$ & & & & & $\square$ & & $N-2$ & $-1 / 2$ & $1 / 2$ & 0 & 0 & 0 & & 0 \\
$A$ & $\Xi$ & & & & & & & -4 & -1 & 0 & 0 & 0 & 0 & & 0 \\
$\bar{Q}_{1}$ & $\square$ & $\square$ & & & & & & 0 & 1 & 0 & 1 & 0 & 0 & & 0 \\
\hline $\bar{Q}_{2}$ & & $\bar{\square}$ & $\square$ & & & & & 0 & -1 & 0 & -1 & 1 & 0 & $\ldots$ & 0 \\
$\bar{Q}_{3}$ & & & $\bar{v}$ & & & & & 0 & 1 & 0 & 1 & -1 & 1 & & 0 \\
$\vdots$ & & & & $\ddots$ & $\square$ & & & 0 & $\vdots$ & 0 & $\vdots$ & & $\vdots$ & & 0 \\
$\bar{Q}_{k}$ & & & & & $\square$ & & $\square$ & 0 & \pm 1 & 0 & \pm 1 & $\mp 1$ & \pm 1 & $\ldots$ & 1 \\
\hline$\Lambda_{1}^{b}$ & & & & & & & & 0 & 0 & 0 & $N$ & 0 & 0 & & 0 \\
$\Lambda_{2}^{b}$ & & & & & & & & 0 & 0 & 0 & 0 & $N$ & 0 & & 0 \\
$\Lambda_{3}^{b}$ & & & & & & & 0 & 0 & 0 & 0 & 0 & $N$ & & 0 \\
$\vdots$ & & & & & & & & $\vdots$ & $\vdots$ & $\vdots$ & $\vdots$ & & & $\ddots$ & 0 \\
$\Lambda_{k}^{b}$ & & & & & & & & 0 & 0 & 0 & 0 & 0 & 0 & & $N$ \\
\hline
\end{tabular}

Table 3. Matter content of the proposed s-confining theory, showing the transformation properties under the gauged $\mathrm{SU}(N)^{k}$ and the $\mathrm{SU}(4)_{L} \times \mathrm{SU}(N)_{R} \times \mathrm{U}(1)_{A} \times \mathrm{U}(1)_{B} \times \mathrm{U}(1)_{R}$ family symmetry. The spurious $\mathrm{U}(1)_{i=1 \ldots k}$ charges are also shown. The alternating $( \pm)$ factors in the $\bar{Q}_{k}$ charges depend on whether $k$ is odd or even: the upper choice corresponds to odd $k$.

\subsection{Infrared operators}

To understand the infrared behavior of the theory, we develop in this section a basis of gauge invariant operators which describe the moduli space and obey anomaly matching conditions. Then in sections 2.2 and 2.3, we find the dynamically generated superpotential and perform some consistency checks.

Let us define a basis for the anomalous $\mathrm{U}(1)$ charges, $\mathrm{U}(1)_{j=1 \ldots k}$, such that the anomaly coefficient $\mathcal{A}\left(G_{i}^{2} \mathrm{U}(1)_{j}\right)$ is zero if and only if $i \neq j$, as shown in table 3. Each $\mathrm{U}(1)_{i}$ is explicitly broken at a scale associated with $\Lambda_{i}$, so that the approximate UV symmetry is broken to

$$
\mathrm{SU}(4)_{L} \times \mathrm{SU}(N)_{R} \times \mathrm{U}(1)_{R} \times \mathrm{U}(1)^{k+2} \longrightarrow \mathrm{SU}(4)_{L} \times \mathrm{SU}(N)_{R} \times \mathrm{U}(1)_{R} \times \mathrm{U}(1)_{A} \times \mathrm{U}(1)_{B}
$$

The $\mathrm{U}(1)_{i}$ charges of the $\Lambda_{i}^{b}$ are determined by the $G^{2} \mathrm{U}(1)$ anomaly coefficients. Note that $b=2 N-1$ for $\Lambda_{1}^{b}$, while $b=2 N$ for $\Lambda_{i \neq 1}^{b}$.

From table 3, it is clear that combinations of the form

$$
\left(\frac{\bar{Q}_{1}^{N} \bar{Q}_{2}^{N}}{\Lambda_{2}^{b}}\right),\left(\frac{\bar{Q}_{2}^{N} \bar{Q}_{3}^{N}}{\Lambda_{3}^{b}}\right), \ldots\left(\frac{\bar{Q}_{k-1}^{N} \bar{Q}_{k}^{N}}{\Lambda_{k}^{b}}\right)
$$

are neutral under all of the symmetries, including the spurious $\mathrm{U}(1)_{i}$. Therefore, the 
dynamically generated superpotential has the form

$$
W_{d} \sim \sum_{p_{2} \ldots p_{k}}\left\{\left(\frac{A^{N-2} Q^{4} \bar{Q}_{1}^{N}}{\Lambda_{1}^{b}}\right)\left(\frac{\bar{Q}_{1}^{N} \bar{Q}_{2}^{N}}{\Lambda_{2}^{b}}\right)^{p_{2}}\left(\frac{\bar{Q}_{2}^{N} \bar{Q}_{3}^{N}}{\Lambda_{3}^{b}}\right)^{p_{3}} \ldots\left(\frac{\bar{Q}_{k-1}^{N} \bar{Q}_{k}^{N}}{\Lambda_{k}^{b}}\right)^{p_{k}}\right\}
$$

for some powers $p_{i}=0,1, \ldots$ for each $i=2,3, \ldots k$. Any such superpotential has an $R$ charge of +2 under all of the possible $\mathrm{U}(1)_{R}$ symmetries. Before we can find the individual terms that appear in $W_{d}$, it is necessary to understand the equations of motion between the infrared operators.

To find a set of gauge invariant operators in the far infrared, let us consider the ordered case $\Lambda_{1} \gg \Lambda_{2} \gg \ldots \gg \Lambda_{k}$. As discussed in section 1.3, $G_{1}$ confinement produces the operators

$$
\begin{aligned}
J_{1} & =\left(Q \bar{Q}_{1}\right), \quad K_{1}=\left(A \bar{Q}_{1}^{2}\right), & Z_{1} & =\left(\bar{Q}_{1}^{N}\right), \\
U_{1} & =\left(A^{m}\right), & V_{1} & =\left(A^{m-1} Q^{2}\right),
\end{aligned}
$$

where $J_{1}$ and $K_{1}$ are charged under $G_{2}$. Although $\mathrm{U}(1)_{1}$ is broken, the $\mathrm{U}(1)_{2} \times \ldots \times \mathrm{U}(1)_{k}$ symmetry is approximately preserved above the scale $\Lambda_{2}$, adding $\mathcal{O}\left(k^{3}\right)$ anomaly coefficients that must be calculated.

This is the benefit of the strategically-defined $\mathrm{U}(1)_{i}$ charges shown in table 3 : the fields $\left\{Q, A, \bar{Q}_{1}\right\}$ are neutral under $\mathrm{U}(1)_{2} \ldots \mathrm{U}(1)_{k}$, and all of these anomaly matching conditions are trivially satisfied. The fields $J_{1}$ and $K_{1}$ transform similarly to $Q$ and $A$ under the non-Abelian symmetries, but their $\mathrm{U}(1)_{B}$ charges are different, as shown in table 4.

At the scale $\Lambda_{2}<\Lambda_{1}$, the $G_{2}$ fields confine to form the following $G_{1} \times G_{2}$ singlets:

$$
\begin{aligned}
& J_{2}=\left(J_{1} \bar{Q}_{2}\right) \quad K_{2}=\left(K_{1} \bar{Q}_{2}^{2}\right) \quad X_{2}=\left(K_{1}^{m} J_{1}\right) \quad Y_{2}=\left(K_{1}^{m-1} J_{1}^{3}\right) \\
& U_{2}=\left(K_{1}^{m}\right) \quad V_{2}=\left(K_{1}^{m-1} J_{1}^{2}\right) \quad \mathcal{W}_{2}=\left(K_{1}^{m-2} J_{1}^{4}\right) \quad Z_{2}=\left(\bar{Q}_{2}^{N}\right) .
\end{aligned}
$$

The fields $J_{2}$ and $K_{2}$ transform under $G_{3}$ as $\square$ and $\boxminus$ respectively.

It is convenient to define the shorthand notation $B_{i}$, where $B_{i}=\left\{U_{i}, V_{i}, \mathcal{W}_{i}\right\}$ for even $N=2 m$, and $B_{i}=\left\{X_{i}, Y_{i}\right\}$ for odd $N=2 m+1$. At scales below $\Lambda_{2}$ and above $\Lambda_{3}$, the intermediate degrees of freedom are $\left\{J_{2}, K_{2}, B_{1}, B_{2}, Z_{1}, Z_{2}, \bar{Q}_{3}, \ldots, \bar{Q}_{k}\right\}$. This set of fields satisfies the anomaly matching conditions for $\mathrm{SU}(4)_{L} \times \mathrm{SU}(N)_{R} \times \mathrm{U}(1)_{A} \times \mathrm{U}(1)_{B} \times \mathrm{U}(1)_{R} \times$ $\mathrm{U}(1)_{3} \times \ldots \times \mathrm{U}(1)_{k}$.

It is straightforward to continue this procedure until all groups including $G_{k}$ have confined, using the following recursive operator definition:

$$
\begin{aligned}
& J_{i}=\left(J_{i-1} \bar{Q}_{i}\right) \quad K_{i}=\left(K_{i-1} \bar{Q}_{i}^{2}\right) \quad X_{i}=\left(K_{i-1}^{m} J_{i-1}\right) \quad Y_{i}=\left(K_{i-1}^{m-1} J_{i-1}^{3}\right) \\
& U_{i}=\left(K_{i-1}^{m}\right) \quad V_{i}=\left(K_{i-1}^{m-1} J_{i-1}^{2}\right) \quad \mathcal{W}_{i}=\left(K_{i-1}^{m-2} J_{i-1}^{4}\right) \quad Z_{i}=\left(\bar{Q}_{i}^{N}\right) .
\end{aligned}
$$

This definition can be applied to $i=1$ as well if we define $J_{0}=Q$ and $K_{0}=A$. Below the scale $\Lambda_{k}$, all of the gauge groups have confined, and the approximate $\mathrm{U}(1)_{i=1 \ldots k}$ symmetries are broken to discrete $\mathbb{Z}_{N}$ groups. The charges under the remaining continuous family symmetries are shown in table 5 . 


\begin{tabular}{|c|c|c|c|c|c|c|c|c|c|c|c|}
\hline & $G_{2}$ & $G_{3}$ & $G_{k}$ & $\mathrm{SU}(4)$ & $\mathrm{SU}(N)$ & $U_{A}$ & $U_{B}$ & $U_{R}$ & $U_{2}$ & $U_{3}$ & $U_{k}$ \\
\hline$J_{1}$ & $\square$ & & & $\square$ & & $N-2$ & $+1 / 2$ & $1 / 2$ & 0 & 0 & 0 \\
\hline$K_{1}$ & $\boxminus$ & & & & & -4 & +1 & 0 & 0 & 0 & 0 \\
\hline $\bar{Q}_{2}$ & $\bar{\square}$ & $\square$ & & & & 0 & -1 & 0 & 1 & 0 & 0 \\
\hline $\bar{Q}_{3}$ & & $\square$ & & & & 0 & +1 & 0 & -1 & 1 & 0 \\
\hline$\vdots$ & & & $\square$ & & & 0 & $\vdots$ & 0 & $\vdots$ & $\vdots$ & 0 \\
\hline $\bar{Q}_{k}$ & & & $\bar{\square}$ & & $\square$ & 0 & \pm 1 & 0 & $\mp 1$ & \pm 1 & $\ldots$ \\
\hline$U_{1}$ & & & & & & $-2 N$ & $-N / 2$ & 0 & 0 & 0 & 0 \\
\hline$V_{1}$ & & & & $\boxminus$ & & 0 & $-N / 2$ & 1 & 0 & 0 & 0 \\
\hline $\mathcal{W}_{1}$ & & & & & & $2 N$ & $-N / 2$ & 2 & 0 & 0 & 0 \\
\hline$X_{1}$ & & & & $\square$ & & $-N$ & $-N / 2$ & $1 / 2$ & 0 & 0 & 0 \\
\hline$Y_{1}$ & & & & $\bar{\square}$ & & $N$ & $-N / 2$ & $3 / 2$ & 0 & 0 & 0 \\
\hline$Z_{1}$ & & & & & & 0 & $N$ & 0 & 0 & 0 & 0 \\
\hline
\end{tabular}

Table 4. Transformation properties of the composite fields in the confined phase of $G_{1}$, in the limit where $G_{2} \times \ldots \times G_{k}$ is weakly gauged. The composite fields $U, V$, and $\mathcal{W}$ exist only if $N$ is even; if $N$ is odd, then they are replaced by $X$ and $Y$.

It must be shown that the basis of infrared operators is large enough to cover the moduli space. For the $\mathrm{SU}(N)^{k}$ gauge group with fields $\left\{A, Q, \bar{Q}_{1}, \ldots, \bar{Q}_{k}\right\}$, the dimension of the moduli space is

$$
\operatorname{dim} M_{0}(k)=\frac{N(N-1)}{2}+4 N+k N^{2}-k\left(N^{2}-1\right)=4 N+\frac{N(N-1)}{2}+k,
$$

while the operator basis $\left\{J_{k}, K_{k} ; B_{1}, \ldots, B_{k} ; Z_{1}, \ldots, Z_{k}\right\}$ has dimension

$$
N_{\text {ops }}=4 N+\frac{1}{2} N(N-1)+9 k
$$

implying that there are $8 k$ complex constraints. By rearranging eq. (2.7) as follows, we can find $8(k-1)$ of the constraint equations:

$$
\begin{aligned}
X_{i} & =\left(K_{i-1}^{m} J_{i-1}\right)=\left(K_{i-2} \bar{Q}_{i-1}^{2}\right)^{m}\left(J_{i-2} \bar{Q}_{i-1}\right)=\left(K_{i-2}^{m} J_{i-2}\right)\left(\bar{Q}_{i-1}^{2 m+1}\right)=X_{i-1} Z_{i-1} \\
Y_{i} & =\left(K_{i-1}^{m-1} J_{i-1}^{3}\right)=\left(K_{i-2} \bar{Q}_{i-1}^{2}\right)^{m-1}\left(J_{i-2} \bar{Q}_{i-1}\right)^{3}=\left(K_{i-2}^{m-1} J_{i-2}^{3}\right)\left(\bar{Q}_{i-1}^{2 m+1}\right)=Y_{i-1} Z_{i-1},
\end{aligned}
$$

for $i=2,3 \ldots k$. Similarly,

$$
U_{i}=U_{i-1} Z_{i-1} \quad V_{i}=V_{i-1} Z_{i-1} \quad \mathcal{W}_{i}=\mathcal{U}_{i-1} Z_{i-1} .
$$

The eight remaining constraints are provided by

$$
X_{k} Z_{k}=K_{k}^{m} J_{k} \quad Y_{k} Z_{k}=K_{k}^{m-1} J_{k}^{3},
$$




\begin{tabular}{|c|cc|ccc|}
\hline & $\mathrm{SU}(4)_{L}$ & $\mathrm{SU}(N)_{R}$ & $U_{A}$ & $U_{B}$ & $U_{R}$ \\
\hline$J_{k}$ & $\square$ & $\square$ & $N-2$ & $\pm 1 / 2$ & $1 / 2$ \\
$K_{k}$ & & $\square$ & -4 & \pm 1 & 0 \\
\hline$U_{\text {odd }}$ & & & $-2 N$ & $-N / 2$ & 0 \\
$V_{\text {odd }}$ & $\square$ & & 0 & $-N / 2$ & 1 \\
$\mathcal{W}_{\text {odd }}$ & & & $2 N$ & $-N / 2$ & 2 \\
\hline$U_{\text {even }}$ & & & $-2 N$ & $+N / 2$ & 0 \\
$V_{\text {even }}$ & $\square$ & & 0 & $+N / 2$ & 1 \\
$\mathcal{W}_{\text {even }}$ & & & $2 N$ & $+N / 2$ & 2 \\
\hline$X_{\text {odd }}$ & $\square$ & & $-N$ & $-N / 2$ & $1 / 2$ \\
$Y_{\text {odd }}$ & $\square$ & & $N$ & $-N / 2$ & $3 / 2$ \\
\hline$X_{\text {even }}$ & $\square$ & & $-N$ & $+N / 2$ & $1 / 2$ \\
$Y_{\text {even }}$ & $\square$ & & $N$ & $+N / 2$ & $3 / 2$ \\
\hline$Z_{\text {odd }}$ & & & 0 & $N$ & 0 \\
$Z_{\text {even }}$ & & & 0 & $-N$ & 0 \\
\hline
\end{tabular}

Table 5. The transformation properties of the composite fields in the fully confined phase of $\mathrm{SU}(N)^{k}$ are shown. The subscript $B_{\text {odd,even }}$ refers to $i=1 \ldots k$, whereas the baryon content $B_{i}=\left\{U_{i}, V_{i}, \mathcal{W}_{i}\right\}$ or $B_{i}=\left\{X_{i}, Y_{i}\right\}$ depends on $N$. The $\mathrm{U}(1)_{B}$ charges of $J_{k}$ and $K_{k}$ are positive if $k$ is odd, and negative if $k$ is even.

or

$$
U_{k} Z_{k}=\operatorname{Pf}\left(K_{k}\right) \quad V_{k} Z_{k}=K_{k}^{m-1} J_{k}^{2} \quad \mathcal{W}_{k} Z_{k}=K_{k}^{m-2} J_{k}^{4} .
$$

It is possible that these classical constraints may be quantum-modified.

Reduced operator basis. The classical constraints for $B_{i>1}$ are potentially problematic, because eqs. (2.11) and (2.12) imply that these operators are redundant: that is, they can be written as products from a smaller operator basis, $\left\{B_{1}, Z_{1}, Z_{2}, \ldots Z_{k}\right\}$, and are therefore not independent degrees of freedom. Excitations of the $B_{i}$ fields above the vacuum acquire $\mathcal{O}\left(\Lambda_{i}\right)$ masses if they do not obey the classical constraints. These massive modes decouple at the scale $\Lambda_{k}$, leaving only the degrees of freedom consistent with the classical (or quantummodified) constraints. Unfortunately, anomaly cancelation depended on the fields $B_{i=2 \ldots k}$ : if these are not true degrees of freedom, then the anomaly matching conditions might not be satisfied.

A solution to this problem can be seen by studying the $X_{\text {odd }}$ and $Y_{\text {even }}$ charges in table 5. Their fermionic components have opposite charges under each of $\mathrm{U}(1)_{A}, \mathrm{U}(1)_{B}$, and $\mathrm{U}(1)_{R}$. When we calculate the anomaly coefficients for each of the mixed and pure $\mathrm{U}(1)$ anomalies, the contributions from each $X_{\text {odd }}$ cancel those from a $Y_{\text {even }}$ field. This is 
also true for the $\mathrm{SU}(4)^{2} \mathrm{U}(1)$ and $\mathrm{SU}(4)^{3}$ anomalies. Therefore, we refer to $X_{\text {odd }}$ and $Y_{\text {even }}$ as an "anomaly neutral pair", indicating that they can be removed without changing any of the anomaly coefficients. Similarly, $X_{\text {even }}$ and $Y_{\text {odd }}$ also form an anomaly neutral pair.

If $k$ is odd, then all of the operators $\left\{X_{2}, Y_{2}, \ldots, X_{k}, Y_{k}\right\}$ can be removed in neutral pairs. Substituting $X_{k}$ and $Y_{k}$ with their equations of motion, eq. (2.13) becomes

$$
\left(X_{1} Z_{1} Z_{2} \ldots Z_{k-1}\right) Z_{k}=K_{k}^{m} J_{k} \quad\left(Y_{1} Z_{1} Z_{2} \ldots Z_{k-1}\right) Z_{k}=K_{k}^{m-1} J_{k}^{3} .
$$

This is not possible if $k$ is even. To remove all the redundant operators, we must also remove a pair $\left\{X_{1}, Y_{\text {even }}\right\}$ or $\left\{X_{\text {even }}, Y_{1}\right\}$, and this is inconsistent: both $X_{1}$ and $Y_{1}$ are necessary to describe the moduli space.

This can be seen if we move away from the origin along the flat direction parameterized by $\left(A^{m} Q\right)$, while keeping $\bar{Q}_{1}=0$. Along this flat direction $X_{1}$ increases, but $X_{\text {even }}=0$. Therefore, $X_{1}$ describes directions on the moduli space that cannot be described by $X_{\text {even }}$. Similarly, by increasing $\left(A^{m-1} Q^{3}\right)$ and fixing $\bar{Q}_{1}=0$, we can see that $Y_{1}$ is just as necessary.

Quantum modification to eq. (2.15) could explain why the odd $k$ and even $k$ situations are different. If $\mathrm{U}(1)_{B}$ is broken in the vacuum, then $\left\{X_{i}, Y_{i}\right\}$ become an anomaly-neutral pair under the remaining symmetries, for any value of $i=1 \ldots k$. Based on $F=N$ SUSY QCD, one would expect the classical relationships involving $\bar{Q}_{i}$ and $\bar{Q}_{i+1}$ to be quantummodified. Specifically, the combination $\left(Z_{i-1} Z_{i}\right)$ has the same spurious $\mathrm{U}(1)_{i}$ charge as $\Lambda_{i}^{b=2 N}$, allowing modifications to equations such as eq. (2.15). For example, the classical $k=4$ constraint for $X_{4} Z_{4}$ might become

$$
X_{1}\left(Z_{1} Z_{2} Z_{3} Z_{4}+\beta_{1} \Lambda_{2}^{b} Z_{3} Z_{4}+\beta_{2} Z_{1} \Lambda_{3}^{b} Z_{4}+\beta_{3} Z_{1} Z_{2} \Lambda_{4}^{b}+\beta_{4} \Lambda_{2}^{b} \Lambda_{4}^{b}\right)=K_{4}^{m} J_{4},
$$

with some as-yet-unknown coefficients $\beta_{i}$. As long as the coefficients are not zero, then the flat direction corresponding to $\left(A^{m} Q\right) \neq 0$ with $\bar{Q}_{1}=0$ now requires some of the $Z_{i \neq 1}$ to have nonzero expectation values. In this $Z_{1}=0, X_{1} \neq 0$ example, eq. (2.16) implies that $\Lambda_{2}^{b}\left(Z_{3} Z_{4}+\Lambda_{4}^{b}\right)=0$, spontaneously breaking $\mathrm{U}(1)_{B}$ even in the limit where $\left\langle X_{1}\right\rangle \gg \Lambda_{k}$. Once $\mathrm{U}(1)_{B}$ is broken in the vacuum, the operators $\left\{J_{4}, K_{4}, X_{1}, Y_{1}, Z_{i=1 \ldots 4}\right\}$ obey the anomaly matching conditions.

A quantum-modified constraint like eq. (2.16) also explains why $\left\{J_{k}, K_{k}, X_{1}, Y_{1}\right.$, $\left.Z_{i=1 \ldots k}\right\}$ is consistent at the origin of moduli space if $k$ is odd. In this case the $Z_{i}=0$ solution remains valid far away from the origin, because every $\Lambda^{b}$ term multiplies at least one $Z$ field. Consider eq. (2.16) with $k=5$ :

$$
\begin{aligned}
K_{5}^{m} J_{5}= & X_{1}\left(Z_{1} Z_{2} Z_{3} Z_{4} Z_{5}+\beta_{1} \Lambda_{2}^{b} Z_{3} Z_{4} Z_{5}+\beta_{2} Z_{1} \Lambda_{3}^{b} Z_{4} Z_{5}+\beta_{3} Z_{1} Z_{2} \Lambda_{4}^{b} Z_{5}+\beta_{4} Z_{1} Z_{2} Z_{3} \Lambda_{5}^{b}\right. \\
& \left.+\beta_{5} Z_{1} \Lambda_{3}^{b} \Lambda_{5}^{b}+\beta_{6} \Lambda_{2}^{b} Z_{3} \Lambda_{5}^{b}+\beta_{7} \Lambda_{2}^{b} \Lambda_{4}^{b} Z_{5}\right) .
\end{aligned}
$$

In this case, the $\left(A^{m} Q\right) \neq 0, \bar{Q}_{i=1 \ldots k}^{N}=0$ flat direction remains on the moduli space for arbitrarily large values of $\left(A^{m} Q\right)$.

This does not mean that $\mathrm{U}(1)_{B}$ is necessarily broken in the vacuum if $k$ is even. Let us fix $Z_{i}=0$ for all $i=1 \ldots k$ to ensure that $\mathrm{U}(1)_{B}$ is not broken at the scale $\Lambda_{i}$. After imposing this constraint, eq. (2.16) becomes

$$
X_{1}=\frac{K_{4}^{m} J_{4}}{\Lambda_{2}^{b} \Lambda_{4}^{b}}
$$


implying that $X_{1}$ is not an IR degree of freedom when $\mathrm{U}(1)_{B}$ is conserved. The same is true for $Y_{1} \Lambda_{2}^{b} \Lambda_{4}^{b}=K_{4}^{m-1} J_{4}^{3}$. In this particular vacuum $X_{1}$ and $Y_{1}$ are redundant operators, and after they are removed from the calculation the $\mathrm{U}(1)_{B}$ anomaly coefficients match the ultraviolet theory.

Theories with even $N$ behave in essentially the same way. Under the exact family symmetries, the operator pairs $\left\{U_{\text {odd }}, \mathcal{W}_{\text {even }}\right\},\left\{U_{\text {even }}, \mathcal{W}_{\text {odd }}\right\}$, and $\left\{V_{\text {odd }}, V_{\text {even }}\right\}$ are anomalyneutral. As in the odd $N$ case, if $k$ is even then it is not possible to remove all the redundant $\left\{U_{i}, V_{i}, \mathcal{W}_{i}\right\}$ operators while preserving the anomaly matching. This leads us to expect that the classical constraint equations

$$
U_{k}=U_{1}\left(Z_{1} Z_{2} \ldots Z_{k-1}\right), \quad V_{k}=V_{1}\left(Z_{1} Z_{2} \ldots Z_{k-1}\right), \quad \mathcal{W}_{k}=\mathcal{W}_{1}\left(Z_{1} Z_{2} \ldots Z_{k-1}\right)
$$

receive quantum modifications of the form

$$
\operatorname{Pf} K_{k}=U_{1}\left(Z_{1} Z_{2} \ldots Z_{k-1}+\ldots+\left(\Lambda_{2}^{b} \Lambda_{4}^{b} \ldots \Lambda_{k-2}^{b}\right) Z_{k-1} Z_{k}+\left(\Lambda_{2}^{b} \Lambda_{4}^{b} \ldots \Lambda_{k}^{b}\right)\right),
$$

if $k$ is even. Either $\mathrm{U}(1)_{B}$ is broken in the vacuum, or the operators $\left\{U_{1}, V_{1}, \mathcal{W}_{1}\right\}$ are not degrees of freedom: in both cases, the IR theory satisfies t' Hooft anomaly matching. Thus, the reduced operator basis describes all infrared degrees of freedom, for both even and odd $N$.

\subsection{Dynamically generated superpotential}

In this section we find a dynamically generated superpotential in the region of parameter space with $\Lambda_{1} \gg \Lambda_{2} \gg \ldots \gg \Lambda_{k}$. We begin by considering how the $W_{d}$ of eq. (1.26) and eq. (1.27) becomes modified at the $G_{2}$ confinement scale. Ignoring the precise relative coefficients between terms,

$$
\begin{aligned}
W_{\text {odd }}^{(1)} & =\frac{1}{\Lambda_{1}^{b}}\left(X_{1} Y_{1} Z_{1}-X_{1} K_{1}^{m-1} J_{1}^{3}-Y_{1} K_{1}^{m} J_{1}\right) \\
W_{\text {even }}^{(1)} & =\frac{1}{\Lambda_{1}^{b}}\left(U_{1} \mathcal{W}_{1} Z_{1}-V_{1}^{2} Z_{1}-U_{1} K_{1}^{m-2} J_{1}^{4}+V_{1} K_{1}^{m-1} J_{1}^{2}-\mathcal{W}_{1} K_{1}^{m}\right) .
\end{aligned}
$$

At the scale $\Lambda_{2}$, we expect $J_{1}$ and $K_{1}$ to confine to form the $B_{2}$ baryons. If we make these replacements in $W^{(1)}$, it becomes

$$
\begin{aligned}
W_{\text {odd }}^{(1)} & =\frac{1}{\Lambda_{1}^{b}}\left(X_{1} Y_{1} Z_{1}-X_{1} Y_{2}-Y_{1} X_{2}\right) \\
W_{\text {even }}^{(1)} & =\frac{1}{\Lambda_{1}^{b}}\left(U_{1} \mathcal{W}_{1} Z_{1}-V_{1}^{2} Z_{1}-\mathcal{W}_{1} U_{2}-U_{1} \mathcal{W}_{2}+V_{1} V_{2}\right) .
\end{aligned}
$$

It is likely that $G_{1}$ confinement changes the holomorphic scale $\Lambda_{2}$ to some new $\widetilde{\Lambda}_{2}$. To find the relationship between $\Lambda_{2}$ and $\widetilde{\Lambda}_{2}$, let us normalize the hadrons to have mass dimension $+1:^{1}$

$$
\widetilde{J}_{1}=\frac{J_{1}}{\Lambda_{1}} \quad \widetilde{K}_{1}=\frac{K_{1}}{\Lambda_{1}^{2}} \quad \widetilde{Z}_{1}=\frac{Z_{1}}{\Lambda_{1}^{N-1}}
$$

\footnotetext{
${ }^{1}$ Even after dividing by these powers of $\Lambda$, it is not necessarily true that the fields are canonically normalized. Corrections in the Kähler potential are likely to require additional normalization.
} 
and similarly for the baryon operators $B_{1}$. The dynamically generated superpotential $W_{2}$ has the form

$$
W^{(2)}=\sum_{\text {contr. }}\left(\frac{\widetilde{K}_{1}^{N-2} \widetilde{J}_{1}^{4} \bar{Q}_{2}^{N}}{\widetilde{\Lambda}_{2}^{b}}\right)=\sum_{\text {contr. }}\left(\frac{K_{1}^{N-2} J_{1}^{4} \bar{Q}_{2}^{N}}{\Lambda_{1}^{2 N} \widetilde{\Lambda}_{2}^{b}}\right) .
$$

From eq. (2.2), symmetry requirements ensure that the superpotential has the form

$$
W^{(2)} \sim \frac{A^{N-2} Q^{4} \bar{Q}_{1}^{N}}{\Lambda_{1}^{b}} \frac{\bar{Q}_{1}^{N} \bar{Q}_{2}^{N}}{\Lambda_{2}^{b}} \longrightarrow \frac{K_{1}^{N-2} J_{1}^{4} \bar{Q}_{2}^{N}}{\Lambda_{1}^{b} \Lambda_{2}^{b}},
$$

allowing $\widetilde{\Lambda}_{2}^{b}$ to be expressed as

$$
\widetilde{\Lambda}_{2}^{2 N-1}=\frac{1}{\Lambda_{1}} \Lambda_{2}^{2 N} .
$$

This expression can also be derived with the same result by matching the gauge couplings at the mass threshold $\Lambda_{1}$. Based on this agreement, we do not expect the superpotential $W_{2}$ to receive modifications of the form

$$
W^{(2)} \rightarrow\left(1+\frac{Z_{1} Z_{2}}{\Lambda_{2}^{b}}+\ldots\right) W^{(2)},
$$

even though such terms are consistent with the family symmetries.

As confinement continues, the products of intermediate mesons $J_{2}$ and $K_{2}$ can be replaced with $G_{3}$ baryons. Each $i=1 \ldots k$ superpotential $W^{(i)}$ becomes

$$
\begin{aligned}
W_{\text {odd }}^{(i<k)} & =\left(\prod_{j=1}^{i} \Lambda_{j}^{b}\right)^{-1}\left(X_{i} Y_{i} Z_{i}-X_{i} Y_{i+1}-Y_{i} X_{i+1}\right) \\
W_{\text {odd }}^{(k)} & =\left(\prod_{j=1}^{k} \Lambda_{j}^{b}\right)^{-1}\left(X_{k} Y_{k} Z_{k}-X_{k} K_{k}^{m-1} J_{k}^{3}-Y_{k} K_{k}^{m} J_{k}\right), \\
W_{\text {even }}^{(i<k)} & =\left(\prod_{j=1}^{i} \Lambda_{j}^{b}\right)^{-1}\left(U_{i} \mathcal{W}_{i} Z_{i}-V_{i}^{2} Z_{i}-\mathcal{W}_{i} U_{i+1}-U_{i} \mathcal{W}_{i+1}+V_{i} V_{i+1}\right) \\
W_{\mathrm{even}}^{(k)} & =\left(\prod_{j=1}^{k} \Lambda_{j}^{b}\right)^{-1}\left(U_{k} \mathcal{W}_{k} Z_{k}-V_{k}^{2} Z_{k}-\mathcal{W}_{k} K_{k}^{m}-U_{k} K_{k}^{m-2} J_{k}^{4}+V_{k} K_{k}^{m-1} J_{k}^{2}\right) .
\end{aligned}
$$

The full superpotential is the sum

$$
W_{d}=\sum_{i=1}^{k} W^{(i)}
$$

Equations of motion. Let us consider equations of motion of the form $\partial W / \partial B_{1}$, where $B_{1}=\left\{U_{1}, V_{1}, \mathcal{W}_{1}, X_{1}, Y_{1}\right\}$ is any of the $G_{1}$ baryons. It is easy to show that these equations are

$$
Y_{2}=Y_{1} Z_{1} \quad X_{2}=X_{1} Z_{1} \quad X_{1} Y_{1}=0
$$


for odd $N$, and

$$
\mathcal{W}_{2}=\mathcal{W}_{1} Z_{1} \quad V_{2}=V_{1} Z_{1} \quad U_{2}=U_{1} Z_{1} \quad U_{1} \mathcal{W}_{1}=V_{1}^{2}
$$

for even $N$. The $\partial W / \partial B_{2}$ equations yield more surprising results: for example,

$$
\frac{\partial W_{d}}{\partial X_{2}}=-\frac{Y_{1}}{\Lambda_{1}^{b}}+\frac{Y_{2} Z_{2}}{\Lambda_{1}^{b} \Lambda_{2}^{b}}=0 \longrightarrow Y_{2} Z_{2}-Y_{3}=Y_{1} \Lambda_{2}^{b}
$$

The classical constraint $Y_{2} Z_{2}=Y_{3}$ is modified, due to the appearance of $X_{2}$ in both $W^{(1)}$ and $W^{(2)}$. For $i=2,3 \ldots(k-1)$, we find

$$
B_{i} Z_{i}=B_{i+1}+\Lambda_{i}^{b} B_{i-1}
$$

The equations of motion $\partial W_{d} / \partial Z_{i}$ are not modified, so that

$$
X_{i} Y_{i}=0, \quad U_{i} \mathcal{W}_{i}=V_{i}^{2}
$$

for all $i$. Finally, the $B_{k}$ equations of motion are

$$
X_{k} Z_{k}=K_{k}^{m} J_{k}+\Lambda_{k}^{b} X_{k-1}, \quad Y_{k} Z_{k}=K_{k}^{m-1} J_{k}^{3}+\Lambda_{k}^{b} Y_{k-1}
$$

for odd $N$, and

$$
U_{k} Z_{k}=K_{k}^{m}+\Lambda_{k}^{b} U_{k-1}, \quad V_{k} Z_{k}=K_{k}^{m-1} J_{k}^{2}+\Lambda_{k}^{b} V_{k-1}, \quad \mathcal{W}_{k} Z_{k}=K_{k}^{m-2} J_{k}^{4}+\Lambda_{k}^{b} \mathcal{W}_{k-1}
$$

for even $N$.

Recall from section 1.3 that each gauge group $\mathrm{SU}(N)_{i}$ has a related $C P$ parameter $\theta_{i}$, which determines the phase of the holomorphic scale $\Lambda_{i}^{b}$. Although $\Lambda^{b} \operatorname{did}$ not appear in the $k=1$ equations of motion, the phases of $\Lambda_{i}^{b}$ do affect the equations of motion in the product group case. The overall phase of $W_{d}$ can still be removed by performing a $\mathrm{U}(1)_{R}$ rotation; however, the relative phases between the $\Lambda_{i}$ may have physical effects.

Armed with these iterative equations of motion, we can rewrite the larger baryons $B_{i>1}$ in terms of $\left\{B_{1}\right\}$ and the $Z_{i}$ fields. For example,

$$
\begin{aligned}
& B_{2}=B_{1} Z_{1} \\
& B_{3}=B_{1}\left(Z_{1} Z_{2}-\Lambda_{2}^{b}\right) \\
& B_{4}=B_{1}\left(Z_{1} Z_{2} Z_{3}-\Lambda_{2}^{b} Z_{3}-Z_{1} \Lambda_{3}^{b}\right) \\
& B_{5}=B_{1}\left(Z_{1} Z_{2} Z_{3} Z_{4}-\Lambda_{2}^{b} Z_{3} Z_{4}-Z_{1} \Lambda_{3}^{b} Z_{4}-Z_{1} Z_{2} \Lambda_{4}^{b}+\Lambda_{2}^{b} \Lambda_{4}^{b}\right) .
\end{aligned}
$$

Our guesses in eqs. (2.16) and (2.17) as to the form of the quantum modification are correct, with $\beta_{i}= \pm 1$ for each coefficient. This process is extended to arbitrary $B_{i}$ in the following way: each classical constraint involving products of the form $\left(Z_{1} Z_{2} \ldots Z_{j}\right)$ is modified by replacing adjacent pairs $\left(Z_{i-1} Z_{i}\right)$ by $\left(-\Lambda_{i}^{b}\right)$, and each possible term is added 
to the product $\left(Z_{1} \ldots Z_{j}\right)$. After making these adjustments, the $k^{\text {th }}$ equations of motion return the following constraints if $k$ is odd:

$$
\begin{aligned}
K_{k}^{m} J_{k} & =X_{1}\left\{\left(Z_{1} \ldots Z_{k}\right)-\Lambda_{2}^{b}\left(Z_{3} \ldots Z_{k}\right)+\ldots+(-1)^{(k-1) / 2}\left(\Lambda_{2}^{b} \Lambda_{4}^{b} \ldots \Lambda_{k-1}^{b}\right) Z_{k}\right\} \\
K_{k}^{m-1} J_{k}^{3} & =Y_{1}\left\{\left(Z_{1} \ldots Z_{k}\right)-\Lambda_{2}^{b}\left(Z_{3} \ldots Z_{k}\right)+\ldots+(-1)^{(k-1) / 2}\left(\Lambda_{2}^{b} \Lambda_{4}^{b} \ldots \Lambda_{k-1}^{b}\right) Z_{k}\right\}, \\
K_{k}^{m} & =U_{1}\left\{\left(Z_{1} \ldots Z_{k}\right)-\Lambda_{2}^{b}\left(Z_{3} \ldots Z_{k}\right)+\ldots+(-1)^{(k-1) / 2}\left(\Lambda_{2}^{b} \Lambda_{4}^{b} \ldots \Lambda_{k-1}^{b}\right) Z_{k}\right\} \\
K_{k}^{m-1} J_{k}^{2} & =V_{1}\left\{\left(Z_{1} \ldots Z_{k}\right)-\Lambda_{2}^{b}\left(Z_{3} \ldots Z_{k}\right)+\ldots+(-1)^{(k-1) / 2}\left(\Lambda_{2}^{b} \Lambda_{4}^{b} \ldots \Lambda_{k-1}^{b}\right) Z_{k}\right\} \\
K_{k}^{m-2} J_{k}^{4} & =\mathcal{W}_{1}\left\{\left(Z_{1} \ldots Z_{k}\right)-\Lambda_{2}^{b}\left(Z_{3} \ldots Z_{k}\right)+\ldots+(-1)^{(k-1) / 2}\left(\Lambda_{2}^{b} \Lambda_{4}^{b} \ldots \Lambda_{k-1}^{b}\right) Z_{k}\right\},
\end{aligned}
$$

or if $k$ is even:

$$
\begin{aligned}
K_{k}^{m} J_{k} & =X_{1}\left\{\left(Z_{1} \ldots Z_{k}\right)+\ldots-(-1)^{\frac{k}{2}}\left(\Lambda_{2}^{b} \ldots \Lambda_{k-2}^{b}\right) Z_{k-1} Z_{k}+(-1)^{\frac{k}{2}}\left(\Lambda_{2}^{b} \Lambda_{4}^{b} \ldots \Lambda_{k}^{b}\right)\right\} \\
K_{k}^{m-1} J_{k}^{3} & =Y_{1}\left\{\left(Z_{1} \ldots Z_{k}\right)+\ldots-(-1)^{\frac{k}{2}}\left(\Lambda_{2}^{b} \ldots \Lambda_{k-2}^{b}\right) Z_{k-1} Z_{k}+(-1)^{\frac{k}{2}}\left(\Lambda_{2}^{b} \Lambda_{4}^{b} \ldots \Lambda_{k}^{b}\right)\right\}, \\
K_{k}^{m} & =U_{1}\left\{\left(Z_{1} \ldots Z_{k}\right)+\ldots-(-1)^{\frac{k}{2}}\left(\Lambda_{2}^{b} \ldots \Lambda_{k-2}^{b}\right) Z_{k-1} Z_{k}+(-1)^{\frac{k}{2}}\left(\Lambda_{2}^{b} \Lambda_{4}^{b} \ldots \Lambda_{k}^{b}\right)\right\} \\
K_{k}^{m-1} J_{k}^{2} & =V_{1}\left\{\left(Z_{1} \ldots Z_{k}\right)+\ldots-(-1)^{\frac{k}{2}}\left(\Lambda_{2}^{b} \ldots \Lambda_{k-2}^{b}\right) Z_{k-1} Z_{k}+(-1)^{\frac{k}{2}}\left(\Lambda_{2}^{b} \Lambda_{4}^{b} \ldots \Lambda_{k}^{b}\right)\right\} \\
K_{k}^{m-2} J_{k}^{4} & =\mathcal{W}_{1}\left\{\left(Z_{1} \ldots Z_{k}\right)+\ldots-(-1)^{\frac{k}{2}}\left(\Lambda_{2}^{b} \ldots \Lambda_{k-2}^{b}\right) Z_{k-1} Z_{k}+(-1)^{\frac{k}{2}}\left(\Lambda_{2}^{b} \Lambda_{4}^{b} \ldots \Lambda_{k}^{b}\right)\right\} .
\end{aligned}
$$

In both cases, the origin of moduli space is a solution to the equations of motion.

As we suggested in section 2.1, if $k$ is even then the $B_{1}$ fields are not independent degrees of freedom when $Z_{i=1 \ldots k}=0$ :

$$
\begin{array}{rlrl}
K_{k}^{m} & =U_{1}(-1)^{\frac{k}{2}}\left(\Lambda_{2}^{b} \Lambda_{4}^{b} \ldots \Lambda_{k}^{b}\right) & K_{k}^{m} J_{k} & =X_{1}(-1)^{\frac{k}{2}}\left(\Lambda_{2}^{b} \Lambda_{4}^{b} \ldots \Lambda_{k}^{b}\right) \\
K_{k}^{m-1} J_{k}^{2} & =V_{1}(-1)^{\frac{k}{2}}\left(\Lambda_{2}^{b} \Lambda_{4}^{b} \ldots \Lambda_{k}^{b}\right) ; & K_{k}^{m-1} J_{k}^{3}=Y_{1}(-1)^{\frac{k}{2}}\left(\Lambda_{2}^{b} \Lambda_{4}^{b} \ldots \Lambda_{k}^{b}\right) . \\
K_{k}^{m-2} J_{k}^{4} & =\mathcal{W}_{1}(-1)^{\frac{k}{2}}\left(\Lambda_{2}^{b} \Lambda_{4}^{b} \ldots \Lambda_{k}^{b}\right)
\end{array}
$$

Therefore, if $\mathrm{U}(1)_{B}$ is a symmetry of the vacuum and $k$ is even, then the $B_{1}$ fields are completely determined by $J_{k}$ and $K_{k}$. After removing the $B_{1}$ fields, the t' Hooft anomaly matching conditions are satisfied. Elsewhere on the moduli space the $B_{1}$ fields may vary independently from $K_{k}$ and $J_{k}, \mathrm{U}(1)_{B}$ is spontaneously broken by $\left\langle Z_{i}\right\rangle \neq 0$, and the anomaly coefficients for the infrared symmetries match the values calculated in the ultraviolet theory.

\subsection{Additional tests}

So far we have restricted our attention to the ordered $\Lambda_{1}>\ldots>\Lambda_{k}$ case to find the dynamically generated superpotential. Due to the holomorphy of the superpotential, changes in the $\Lambda_{i}$ hierarchy should not alter the form of the superpotential. In this section we test this supposition by considering the $\Lambda_{1} \ll \Lambda_{i \neq 1}$ case. In this limit the $\mathrm{SU}(N)^{k}$ model reduces to an $\mathrm{SU}(N)^{k-1}$ extension to $F=N$ SUSY QCD which has been studied by Chang and Georgi [11]. 
As $\Lambda_{1} \rightarrow 0$, the $A$ and $Q$ fields decouple from the strongly coupled $\bar{Q}_{i}$. Chang and Georgi find that the infrared operators involving only $\bar{Q}_{i}$ obey the following constraints:

$$
\begin{aligned}
\operatorname{det}\left(\bar{Q}_{1} \bar{Q}_{2}\right) & =Z_{1} Z_{2}-\Lambda_{2}^{b} \\
\operatorname{det}\left(\bar{Q}_{1} \bar{Q}_{2} \bar{Q}_{3}\right) & =Z_{1} Z_{2} Z_{3}-\Lambda_{2}^{b} Z_{3}-Z_{1} \Lambda_{3}^{b} \\
\operatorname{det}\left(\bar{Q}_{1} \bar{Q}_{2} \bar{Q}_{3} \bar{Q}_{4}\right) & =Z_{1} Z_{2} Z_{3} Z_{4}-\Lambda_{2}^{b} Z_{3} Z_{4}-Z_{1} \Lambda_{3}^{b} Z_{4}-Z_{1} Z_{2} \Lambda_{4}^{b}+\Lambda_{2}^{b} \Lambda_{4}^{b},
\end{aligned}
$$

and so on. This is exactly the same form we derived for $B_{i \geq 2}$ in section 2.2. At scales above $\mathcal{O}\left(\Lambda_{1}\right)$ but below $\Lambda_{i>1}$, the $G_{1}$ charged degrees of freedom include $A, Q$, and $M=$ $\left(\bar{Q}_{1} \bar{Q}_{2} \ldots \bar{Q}_{k}\right)$. Let us define the mass-normalized field $\widetilde{M}$,

$$
\widetilde{M}=\frac{\left(\bar{Q}_{1} \bar{Q}_{2} \ldots \bar{Q}_{k}\right)}{\Lambda_{2} \Lambda_{3} \ldots \Lambda_{k}}
$$

and let the fields $\{A, Q, \widetilde{M}\}$ confine under $G_{1}$, producing

$$
J_{k}=Q \widetilde{M}, \quad K_{k}=A \widetilde{M}^{2}, \quad Z_{M}=\operatorname{det}(\widetilde{M}),
$$

and the baryons $B_{1}=\left\{U_{1}, V_{1}, \mathcal{W}_{1} ; X_{1}, Y_{1}\right\}$ as defined in section 2.1. The dynamically generated superpotential is

$$
\begin{aligned}
W_{\text {odd }} & =\frac{X_{1} Y_{1} Z_{M}-X_{1} K_{k}^{m-1} J_{k}^{3}-Y_{1} K_{k}^{m} J_{k}}{\widetilde{\Lambda}_{1}^{b}} \\
W_{\text {even }} & =\frac{\left(U_{1} \mathcal{W}_{1}-V_{1}^{2}\right) Z_{M}-U_{1} K_{k}^{m-2} J_{k}^{4}+V_{1} K_{k}^{m-1} J_{k}^{2}-\mathcal{W}_{1} K_{k}^{m}}{\widetilde{\Lambda}_{1}^{b}} .
\end{aligned}
$$

The effective scale $\widetilde{\Lambda}_{1}^{b}$ contains a product of $\left(\bar{Q}_{1}^{N} \ldots \bar{Q}_{k}^{N}\right)$ and $\Lambda_{2}^{b} \ldots \Lambda_{k}^{b}$, so that the superpotential is invariant under the spurious symmetries.

There is also a quantum modified constraint

$$
Z_{M}=\operatorname{det} \widetilde{M}=\left(Z_{1} \ldots Z_{k}\right)-\Lambda_{2}^{b}\left(Z_{3} \ldots Z_{k}\right)+\{\text { all other contractions }\} .
$$

If we use a Lagrange multiplier $\lambda$, eq. (2.56) follows from the superpotential

$$
W_{d}^{\prime}=\lambda\left\{Z_{M}-\left(Z_{1} \ldots Z_{k}\right)+(\text { all contractions })\right\}
$$

After replacing $Z_{M}$ with $\left\{Z_{i}\right\}$, the equations of motion are identical to eqs. (2.46) and (2.47), suggesting that there is no phase transition in the parameter space.

Notice that the equations of motion from $Z_{M}$ also determine a vacuum solution for $\lambda$ :

$$
\begin{aligned}
& \frac{\partial W_{\text {odd }}}{\partial Z_{M}}=\frac{X_{1} Y_{1}}{\widetilde{\Lambda}_{1}^{b}}+\lambda=0 \\
& \frac{\partial W_{\text {even }}}{\partial Z_{M}}=\frac{U_{1} \mathcal{W}_{1}-V_{1}^{2}}{\widetilde{\Lambda}_{1}^{b}}+\lambda=0 .
\end{aligned}
$$


Thus, the Lagrange multiplier can be treated as a new redundant baryon operator, which should be integrated out along with the other redundant fields.

Finally, let us consider regions of parameter space in which $\Lambda_{1}$ is neither the largest nor the smallest confinement scale. In these cases the redundant operators include a mix of $B_{i}$ and $Z_{i j}$, all of which produce the same equations of motion in the reduced operator basis. For any arrangement, at the last confinement scale $\Lambda_{f}$ there is a dynamically generated superpotential of the form

$$
W^{(f)} \sim \frac{\widetilde{K}_{f}^{N-2} \widetilde{J}_{f}^{4} \widetilde{M}^{N}}{\widetilde{\Lambda}_{f}^{b}},
$$

where $J_{f}, K_{f}$, and $M$ are such that

$$
\left(J_{f} M\right)=\left(Q \bar{Q}_{1} \ldots \bar{Q}_{f}\right)\left(\bar{Q}_{f+1} \ldots \bar{Q}_{k}\right)=J_{k}, \quad\left(K_{f} M^{2}\right)=\left(A \bar{Q}_{1}^{2} \ldots \bar{Q}_{f}^{2}\right)\left(\bar{Q}_{f+1} \ldots \bar{Q}_{k}^{2}\right)^{2}=K_{k}
$$

and where $\left\{\widetilde{J}_{f}, \widetilde{K}_{f}, \widetilde{M}\right\}$ are normalized to have mass dimension +1 . Under the remaining gauged $G_{f}$, these fields satisfy the index condition for s-confinement, $\sum_{j} \mu_{j}-\mu_{G}=2$, and there is a dynamically generated superpotential. Lagrange multipliers $\lambda_{i}$ enforce the constraint between the operators $\operatorname{det}\left(\bar{Q}_{i} \ldots \bar{Q}_{j}\right)$ and $\left\{Z_{i} \ldots Z_{j}\right\}$, and the equations of motion provide a relationship between $\lambda_{i}$ and the other hadrons. After replacing the redundant operators with their equations of motion, we find that the constraints relating $\left\{J_{k}, K_{k}\right\}$ to $\left\{B_{1}, Z_{i}\right\}$ are unchanged.

Flow. It is a necessary condition for s-confining theories that their description in terms of gauge-invariants is valid in the Higgs phase, when some fields acquire large expectation values and spontaneously break the gauge group to a subgroup. If the low-energy theory does not s-confine, then the original theory cannot be s-confining either. This is the "flow requirement" of [4], which we use in this section to test the $\mathrm{SU}(N)^{k}$ theory.

In the $\left\langle J_{k}\right\rangle_{j}^{i} \gg \Lambda$ vacuum with $\left\langle A_{\alpha \beta}\right\rangle=0$, the $\mathrm{SU}(N)^{k}$ group is broken to $\mathrm{SU}(N-$ $1)^{k}$ in the classical limit. This requires a nonzero $\left(\bar{Q}_{i}\right)_{\beta}^{\alpha}$ for every $\bar{Q}_{i}$, which break each gauged $\mathrm{SU}(N)_{i}$ to $\mathrm{SU}(N-1)_{i}$. The $\mathrm{SU}(N)_{i} \times \mathrm{SU}(N)_{i+1}$ bifundamentals $\bar{Q}_{i}$ decompose into $\mathrm{SU}(N-1) \times \mathrm{SU}(N-1)$ representations as follows:

$\mathrm{SU}(N) \times \mathrm{SU}(N) \rightarrow \mathrm{SU}(N-1) \times \mathrm{SU}(N-1):(\bar{\square}, \square) \longrightarrow(\bar{\square}, \square) \oplus(\bar{\square}, \mathbf{1}) \oplus(\mathbf{1}, \square) \oplus(\mathbf{1}, \mathbf{1})$.

The $(2 N-1)$ broken generators of each gauge group $G_{i \neq 1}$ "eat" the combination $\square+\bar{\square}+\mathbf{1}$ from $\bar{Q}_{i-1}$ and $\bar{Q}_{i}$ to create $(2 N-1)$ massive gauge superfields, leaving behind the $(\bar{\square} \square)$ bifundamental fields.

The $G_{1}$ group behaves somewhat differently: its broken generators "eat" the $(\bar{\square}, \mathbf{1})$ part of $\bar{Q}_{1}$ and a linear combination of the $\square$ superfields $Q_{i=1 \ldots 4}$. Under $\operatorname{SU}(N-1)_{1}$ the $\square$ field decomposes as $(\boxminus \oplus \square)$, so that the "eaten" $Q$ field is replaced by a component of $A$. After removing the massive superfields, the $\mathrm{SU}(N-1)_{1}$ charged matter is $A^{\prime}+4 Q^{\prime}+(N-1) \bar{Q}_{1}^{\prime}$. The overall effect of $\left\langle J_{k}\right\rangle \gg \Lambda$ on the $\mathrm{SU}(N)^{k}$ model is to replace $N$ with $N-1$. 
Now let us consider the limit where $\left\langle A_{\alpha \beta}\right\rangle \gg \Lambda$ and $\langle J\rangle=0$. In the even $N=2 m$ case with $\left\langle U_{1}=\operatorname{Pf} A\right\rangle \gg \Lambda_{1}, \mathrm{SU}(2 m)_{1}$ is broken to $\mathrm{Sp}(2 m)_{1}$ and $\boxminus$ decomposes into $\square_{\mathrm{Sp}} \oplus \mathbf{1}$. Here $\boxminus_{\mathrm{Sp}}$ is the $\left(2 m^{2}-m-1\right)$ dimensional representation of $\operatorname{Sp}(2 m)$. There are also $\left(2 m^{2}-m-1\right)$ broken $\mathrm{SU}(2 m)$ generators, so the superfield $A^{\prime}=\bigoplus_{\mathrm{Sp}}$ is eaten.

The fields $Q$ and $\bar{Q}_{i}$ are not directly affected by $\langle\operatorname{Pf} A\rangle$ : however, as $\operatorname{Sp}(2 m)$ has no complex representations, $Q$ and $\bar{Q}_{1}$ are effectively $(2 m+4)$ quarks charged in the $\square$ representation of $\operatorname{Sp}(2 m)$. This theory is known to s-confine [22]. It is likely that the $\mathrm{Sp}(2 m) \times \mathrm{SU}(2 m)^{k-1}$ product group theory is also s-confining: we explore this possibility in section 3.2 .

In the case where $N$ is odd, an expectation value $\left\langle X_{1}\right\rangle=\left\langle A^{m} Q\right\rangle \gg \Lambda$ breaks $\mathrm{SU}(2 m+1)$ to $\mathrm{Sp}(2 m)$ instead. Aside from a few extra singlets and massive gauge bosons, there is little difference between the odd $N$ and even $N$ cases: the infrared theory is $\mathrm{Sp}(2 m) \times \mathrm{SU}(2 m)^{k-1}$.

Conclusion. Our product group extension to the $A+4 Q+N \bar{Q}$ model exhibits the behavior required for an s-confining theory. The set of gauge invariant operators $\left\{J_{k}, K_{k}, B_{1}, Z_{1 \ldots k}\right\}$ satisfies the t' Hooft anomaly matching conditions; the origin remains on the quantum moduli space, so the theory can confine without breaking chiral symmetry; and there is a dynamically generated superpotential. Furthermore, the operators $\left\{J_{k}, K_{k}, B_{1}, Z_{1 \ldots k}\right\}$ provide a smooth description of the entire moduli space: there is no gauge invariant order parameter to distinguish the confined and Higgs phases. By considering the flow along flat directions, we have also found another product group extension to an s-confining theory, $\mathrm{Sp}(2 m) \times \mathrm{SU}(N)^{k-1}$.

\section{Other s-confining theories}

In the previous section we find strong evidence that the product group extension to the $A+4 Q+N \bar{Q}$ model is s-confining. In this section we consider the follow-up question: how many other s-confining models can be extended into product groups? We have already suggested that $\operatorname{Sp}(2 m)$ with $(2 m+4) \square$ can be extended into an $\operatorname{Sp}(2 m) \times \operatorname{SU}(N)^{k-1}$ product group model. If this theory is not s-confining, then the $\mathrm{SU}(N)^{k} A+4 Q+N \bar{Q}$ model is not s-confining either. We discuss the behavior of this theory in section 3.2.

There are also additional possibilities for the $A+4 Q+N \bar{Q}$ model in the case where $N=4$. In this special case the entire $\mathrm{SU}(4)_{L} \times \mathrm{SU}(N)_{R}$ family symmetry can be gauged: we consider whether or not such theories are s-confining in section 3.1. In sections 3.3 and 3.4 we discuss the other s-confining theories in [5] with family symmetries large enough to accommodate a gauged $\mathrm{SU}(N)$ subgroup. This includes SUSY QCD with $F=N+1$ flavors, and $\operatorname{Sp}(2 m)$ with $(\square+6 \square)$ matter for $m=2$ and $m=3$. We show that some of these theories are not s-confining.

Due to the lack of an index constraint on the matter content, it is difficult to conduct a systematic search for new s-confining product groups. We have seen in the $A+4 Q+N \bar{Q}$ model that $G_{1}$ confinement increases the index sum of the $G_{2}$ charged matter by +2 , but 


\begin{tabular}{|c|c|c|c|c|c|c|c|c|c|c|}
\hline & $\mathrm{SU}(4)_{L}$ & $G_{2}$ & $G_{1}$ & $G_{0}$ & $\widetilde{G}_{1}$ & $\widetilde{G}_{2}$ & $\mathrm{SU}(4)_{R}$ & $U_{A}$ & $U_{B}$ & $U_{R}$ \\
\hline$Q_{2}$ & $\bar{\square}$ & $\square$ & & & & & & 1 & 0 & 0 \\
\hline$Q_{1}$ & & $\bar{\square}$ & $\square$ & & & & & -1 & 0 & 0 \\
\hline$Q_{0}$ & & & $\bar{\square}$ & $\square$ & & & & 1 & 0 & 0 \\
\hline$A$ & & & & $\boxminus$ & & & & -2 & -2 & 1 \\
\hline $\bar{Q}_{0}$ & & & & $\bar{\square}$ & $\square$ & & & 0 & 1 & 0 \\
\hline $\bar{Q}_{1}$ & & & & & $\bar{\square}$ & $\square$ & & 0 & -1 & 0 \\
\hline $\bar{Q}_{2}$ & & & & & & $\bar{\square}$ & $\square$ & 0 & 1 & 0 \\
\hline
\end{tabular}

Table 6. Above, the original s-confining theory $A+4\left(Q_{0}+\bar{Q}_{0}\right)$ is extended on the left and right by gauging $G_{L}^{2} \times \widetilde{G}_{R}^{2}$ and adding the $Q_{i}$ and $\bar{Q}_{i}$ fields to cancel the anomalies. To extend the model beyond $\ell=r=2$, more quarks $Q_{i}$ and $\bar{Q}_{j}$ can be added with alternating $\mathrm{U}(1)_{A}$ and $\mathrm{U}(1)_{B}$ charges.

other confining theories tend to change the index sum by varying amounts. Therefore, the list of theories considered in this section is presumably incomplete.

We restrict our attention to s-confining models which can be extended by gauging a subgroup of the family symmetries and adding bifundamental fields. Our goal is to determine whether product group s-confinement is possible in each model, based on the index constraint after confinement. This is sufficient to show which of the product group extensions are obviously not s-confining. A more detailed analysis is appropriate for the theories which pass this test.

\subsection{Special case: $\mathrm{SU}(4)$}

In this section, we extend the $N=4 A+4 Q+N \bar{Q}$ model by gauging $\mathrm{SU}(4)_{L}^{\ell} \times G_{0} \times \mathrm{SU}(4)_{R}^{r}$ for some $\ell$ and $r$. Here $G_{0}$ is the $\mathrm{SU}(4)$ gauge group containing the $\boxminus+4(\square+\square)$ matter, and every other gauged SU(4) contains four flavors of $(\square+\square)$. It is convenient to relabel the hadrons to reflect the $Q \leftrightarrow \bar{Q}$ symmetry of the matter content of the $A+4 Q+4 \bar{Q}$ model:

$$
M=Q \bar{Q}, \quad \bar{K}=A \bar{Q}^{2}, \quad K=A Q^{2}, \quad U=A^{2}, \quad Z=Q^{4}, \quad \bar{Z}=\bar{Q}^{4} .
$$

A convenient redefinition of the $\mathrm{U}(1)_{A} \times \mathrm{U}(1)_{B} \times \mathrm{U}(1)_{R}$ charges is shown in table 6 , for $\ell=r=2$.

After extending the model in this way, the model has a "left-right" symmetry which simplifies many of the calculations in this section:

$$
\ell \leftrightarrow r, \quad G_{i} \leftrightarrow \widetilde{G}_{i}, \quad \Lambda_{i} \leftrightarrow \bar{\Lambda}_{i}, \quad \mathrm{SU}(4)_{L} \leftrightarrow \mathrm{SU}(4)_{R}, \quad \mathrm{U}(1)_{A} \leftrightarrow \mathrm{U}(1)_{B}, \quad Q_{i} \leftrightarrow \bar{Q}_{i}
$$

Above, $\Lambda_{i}$ corresponds to the group $G_{i}$, while $\bar{\Lambda}_{i}$ is the confinement scale of the group $\widetilde{G}_{i}$. The group $G_{0} \times \mathrm{U}(1)_{R}$ and the field $A$ are invariant under the discrete transformation. 
Infrared operators. Based on our understanding of the $(\ell=0, r=k-1)$ models developed in the previous section and the vectorlike nature of the $G_{0}$-charged fields, we can guess the form of the gauge-invariant operators which describe the moduli space:

$$
\mathcal{F} \equiv\left\{\begin{aligned}
U_{1} & =A^{2} & M_{\ell r} & =\left(Q_{\ell} \ldots Q_{1} Q_{0} \bar{Q}_{0} \bar{Q}_{1} \ldots \bar{Q}_{r}\right) \\
Z_{i} & =Q_{i}^{4} & K_{\ell} & =\left(Q_{\ell}^{2} \ldots Q_{0}^{2} A\right) \\
\bar{Z}_{j} & =\bar{Q}_{j}^{4}, & \bar{K}_{r} & =\left(A \bar{Q}_{0}^{2} \ldots \bar{Q}_{r}^{2}\right)
\end{aligned}\right\},
$$

for $i=0,1, \ldots, \ell$ and $j=0,1, \ldots, r$.

Only under certain conditions do we expect the basis $\mathcal{F}$ to obey the anomaly matching conditions for the family symmetries listed in table 6 . We have already seen that in the $(\ell=0, r=k-1)$ models with even $k$, some of the operators in $\mathcal{F}$ become redundant in the $\mathrm{U}(1)_{B}$ preserving vacuum. If this pattern continues in the $(\ell, r)$ models with $\ell \neq 0$ and $r \neq 0$, then we would expect that the set $\mathcal{F}$ obeys the anomaly matching conditions only if $\ell$ and $r$ are even. If either $\ell$ or $r$ is odd, we expect that some operators in $\mathcal{F}$ become redundant if $\mathrm{U}(1)_{A} \times \mathrm{U}(1)_{B}$ is preserved in the vacuum.

For a given $(\ell, r)$, the number of infrared operators is given by

$$
\operatorname{dim} \mathcal{F}=1+(\ell+1)+(r+1)+4^{2}+\frac{4(3)}{2}+\frac{4(3)}{2}=\ell+r+31,
$$

while the dimension of the classical moduli space is

$$
\operatorname{dim} M_{0}=(\ell+1) 4^{2}+\frac{4(3)}{2}+(r+1) 4^{2}-(\ell+1+r)\left(4^{2}-1\right)=\ell+r+23 .
$$

This implies that there should exist $N_{\text {con }}=8$ constraint equations.

Equations of motion. It is easiest to derive the equations of motion in the case where $G_{0}$ confines last. The groups $G_{1} \times \ldots \times G_{\ell}$ and $\widetilde{G}_{1} \times \ldots \times \widetilde{G}_{r}$ confine separately to form the mesons $M_{L}=\left(Q_{0} \ldots Q_{\ell}\right)$ and $M_{R}=\left(\bar{Q}_{0} \ldots \bar{Q}_{r}\right)$, the baryons $Z_{i=0 \ldots \ell}$ and $\bar{Z}_{j=0 \ldots r}$, and some larger baryon operators with quantum-modified constraints. The charges of $M_{L}$ and $M_{R}$ are shown in table 7 . In the limit where $\Lambda_{0}$ is small, the theory reduces to two copies of $F=N$ SUSY QCD with product group extensions. According to [11], the fields obey the following constraints:

$$
\begin{aligned}
\operatorname{det} M_{L} & =\left(Z_{0} Z_{1} \ldots Z_{\ell}\right)-\Lambda_{1}^{b}\left(Z_{2} \ldots Z_{\ell}\right)-\ldots-\left(Z_{0} \ldots Z_{\ell-2}\right) \Lambda_{\ell}^{b}+\ldots \\
\operatorname{det} M_{R} & =\left(\bar{Z}_{0} \bar{Z}_{1} \ldots \bar{Z}_{r}\right)-\bar{\Lambda}_{1}^{b}\left(\bar{Z}_{2} \ldots \bar{Z}_{r}\right)-\ldots-\left(\bar{Z}_{0} \ldots \bar{Z}_{r-2}\right) \bar{\Lambda}_{r}^{b}+\ldots
\end{aligned}
$$

If $\ell$ is odd-valued, then the sum of neighbor contractions includes a constant term, $\left(\Lambda_{1}^{b} \Lambda_{3}^{b} \ldots \Lambda_{\ell}^{b}\right)$; if $\ell$ is even, then all terms include some power of $Z_{i}$. The same relationship holds for $r$ and $\operatorname{det} M_{R}$. As in the $\mathrm{SU}(N)^{k}$ models, we expect that the distinction between even and odd $\ell$ and $r$ determines which of the operators in $\mathcal{F}$ are redundant when $\mathrm{U}(1)_{A}$ and $\mathrm{U}(1)_{B}$ are conserved in the vacuum.

When $G_{0}$ confines, $\left\{M_{L}, A, M_{R}\right\}$ form the following hadrons:

$$
\begin{aligned}
U_{1} & =A^{2} & M_{\ell r} & =\left(M_{L} M_{R}\right) \\
Z_{L} & =\operatorname{det} M_{L} & K_{\ell} & =\left(A M_{L}^{2}\right) \\
Z_{R} & =\operatorname{det} M_{R} & \bar{K}_{r} & =\left(A M_{R}^{2}\right),
\end{aligned}
$$




\begin{tabular}{|c|c|c|c|ccc|}
\hline & $\mathrm{SU}(4)_{L}$ & $G_{0}$ & $\mathrm{SU}(4)_{R}$ & $U_{A}$ & $U_{B}$ & $U_{R}$ \\
\hline$M_{L}$ & $\square$ & $\square$ & & $\{0,1\}$ & 0 & 0 \\
$A$ & & $\square$ & & -2 & -2 & 1 \\
$M_{R}$ & & $\square$ & $\square$ & 0 & $\{0,1\}$ & 0 \\
\hline
\end{tabular}

Table 7. All gauge groups except $G_{0}$ have confined, leaving $M_{L}$ and $M_{R}$. The $\{0,1\}$ charges of $M_{L}$ and $M_{R}$ correspond to the cases where $\ell$ and $r$ are odd or even, respectively. Not shown are the baryons $Z_{i}$ and $\bar{Z}_{j}$, which do not transform under the non-Abelian symmetries.

\begin{tabular}{|c|cc|cc|cc|c|}
\hline & $\mathrm{SU}(4)_{L}$ & $\mathrm{SU}(4)_{R}$ & $U_{A}^{\text {odd } \ell}$ & $U_{A}^{\text {even } \ell}$ & $U_{B}^{\text {odd } r}$ & $U_{B}^{\text {even } r}$ & $U_{R}$ \\
\hline$K_{\ell}$ & $\square$ & & -2 & 0 & -2 & -2 & 1 \\
$M_{\ell r}$ & $\square$ & $\square$ & 0 & 1 & 0 & 1 & 0 \\
$\bar{K}_{r}$ & & $\square$ & -2 & -2 & -2 & 0 & 1 \\
\hline$U_{1}$ & & & -4 & -4 & -4 & -4 & 2 \\
$Z_{\text {even } i}$ & & & +4 & +4 & 0 & 0 & 0 \\
$Z_{\text {odd } i}$ & & & -4 & -4 & 0 & 0 & 0 \\
$\bar{Z}_{\text {even } j}$ & & & 0 & 0 & +4 & +4 & 0 \\
$\bar{Z}_{\text {odd } j}$ & & & 0 & 0 & -4 & -4 & 0 \\
\hline
\end{tabular}

Table 8. After all of the gauge groups confine, the infrared degrees of freedom are described by the hadrons shown above. Their $\mathrm{U}(1)_{A}$ and $\mathrm{U}(1)_{B}$ charges depend on $\ell$ and $r$, respectively.

with the dynamically-generated superpotential

$$
W_{d} \sim \frac{A^{2} \widetilde{M}_{L}^{4} \widetilde{M}_{R}^{4}}{\widetilde{\Lambda}_{0}^{b}} \sim \frac{U_{1} Z_{L} Z_{R}-Z_{R} K_{\ell}^{2}-Z_{L} \bar{K}_{r}^{2}-U_{1} M_{\ell r}^{4}+K_{\ell} M_{\ell r}^{2} \bar{K}_{r}}{\widetilde{\Lambda}_{0}^{b}\left(\Lambda_{1} \ldots \Lambda_{\ell}\right)^{4}\left(\bar{\Lambda}_{1} \ldots \bar{\Lambda}_{r}\right)^{4}},
$$

for some $\widetilde{\Lambda}_{0}^{b}$ consistent with the anomalous symmetries. We show the charges of the composite fields in table 8 .

The equations of motion from $U_{1}, K_{\ell}$, and $Z_{L}$ produce the following constraints:

$$
\begin{aligned}
& \operatorname{det} M_{\ell r}=Z_{L} Z_{R} \quad K_{\ell} Z_{R}=M_{\ell r}^{2} \bar{K}_{r} \quad \operatorname{Pf} \bar{K}_{r}=U_{1} Z_{R} \\
& U_{1} M^{3}=K_{\ell} M \bar{K}_{r}, \quad \bar{K}_{r} Z_{L}=K_{\ell} M_{\ell r}^{2}, \quad \operatorname{Pf} K_{\ell}=U_{1} Z_{L} .
\end{aligned}
$$

These equations are not all independent, but contain $N_{\text {cons }}=8$ independent constraints.

If we introduce Lagrange superfields $\lambda_{L}$ and $\lambda_{R}$, the quantum modified constraints relating $\left\{Z_{L}, Z_{R}\right\}$ to $\left\{Z_{i}, \bar{Z}_{j}\right\}$ as a superpotential:

$$
\begin{aligned}
& W_{L}=\lambda_{L}\left(Z_{L}-\left(Z_{0} Z_{1} \ldots Z_{\ell}\right)+\Lambda_{1}^{b}\left(Z_{2} \ldots Z_{\ell}\right)+\ldots+\left(Z_{0} \ldots Z_{\ell-2}\right) \Lambda_{\ell}^{b}+\ldots\right) \\
& W_{R}=\lambda_{R}\left(Z_{R}-\left(\bar{Z}_{0} \bar{Z}_{1} \ldots \bar{Z}_{r}\right)+\bar{\Lambda}_{1}^{b}\left(\bar{Z}_{2} \ldots \bar{Z}_{r}\right)+\ldots+\left(\bar{Z}_{0} \ldots \bar{Z}_{r-2}\right) \bar{\Lambda}_{r}^{b}+\ldots\right) .
\end{aligned}
$$

Redundant operators. In this section we use the equations of motion to study the operator basis $\mathcal{F}$. In the $\mathrm{U}(1)_{A}$ preserving vacuum with $\left\langle Z_{i}\right\rangle=0$, the expectation value of 
$Z_{L}$ depends heavily on whether $\ell$ is even or odd. If $\ell$ is even, then $Z_{L} \approx 0$; if $\ell$ is odd, then $Z_{L} \approx\left(\Lambda_{1}^{b} \Lambda_{3}^{b} \ldots \Lambda_{\ell}^{b}\right) \gg 0$. The same pattern holds for $r$ and $\bar{Z}_{j}$ when $\mathrm{U}(1)_{B}$ is preserved.

It is simplest to consider the case in which both $\ell$ and $r$ are even. Expanding about the $Z_{i}=\bar{Z}_{j}=0$ vacuum to first order in $Z_{i}$ and $\bar{Z}_{j}$, we find that every term in eq. (3.10) contains a product of at least two fields, so that none of the operators in the set $\mathcal{F}$ are redundant. This is consistent with the fact that all of the anomaly coefficients from $\mathrm{SU}(4)_{L} \times \mathrm{SU}(4)_{R} \times \mathrm{U}(1)_{A} \times \mathrm{U}(1)_{B} \times \mathrm{U}(1)_{R}$ match the ultraviolet theory when $r$ and $\ell$ are even.

This is not true if $\ell$ is odd. In this case the equations of motion for $\bar{K}_{r} Z_{L}$ and $U_{1} Z_{L}$ can be rewritten as

$$
\bar{K}_{r}=\frac{K_{\ell} M_{\ell r}^{2}}{\left(\Lambda_{1}^{b} \Lambda_{3}^{b} \ldots \Lambda_{\ell}^{b}\right)}, \quad U_{1}=\frac{\operatorname{Pf} K_{\ell}}{\left(\Lambda_{1}^{b} \Lambda_{3}^{b} \ldots \Lambda_{\ell}^{b}\right)}
$$

near the $\mathrm{U}(1)_{A} \times \mathrm{U}(1)_{B}$ preserving vacuum. Similarly, the equation of motion for $\operatorname{det} M_{\ell r}$ becomes

$$
\bar{Z}_{0}\left(\bar{\Lambda}_{2}^{b} \bar{\Lambda}_{4}^{b} \ldots \bar{\Lambda}_{r}^{b}\right)+\bar{\Lambda}_{1}^{b} \bar{Z}_{2}\left(\bar{\Lambda}_{4}^{b} \ldots \bar{\Lambda}_{r}^{b}\right)+\ldots+\left(\bar{\Lambda}_{1}^{b} \bar{\Lambda}_{3}^{b} \ldots \bar{\Lambda}_{r-1}^{b}\right) \bar{Z}_{r}=\frac{\operatorname{det} M_{\ell r}}{\left(\Lambda_{1}^{b} \Lambda_{3}^{b} \ldots \Lambda_{\ell}^{b}\right)},
$$

which can be recast into a linear constraint equation for any one of the $\bar{Z}_{\text {even }}$ fields. Taken together, eqs. (3.13) and (3.14) imply that the operators $\left\{\bar{K}_{r}, U_{1}, \bar{Z}_{\text {even }}\right\}$ should be removed in the $\mathrm{U}(1)_{A} \times \mathrm{U}(1)_{B}$ preserving vacuum if $\ell$ is odd and $r$ is even. In the even $\ell$, odd $r$ case it is the operators $\left\{K_{\ell}, U_{1}, Z_{\text {even }}\right\}$ which become redundant, and $Z_{R}$ rather than $Z_{L}$ remains large in the $\bar{Z}_{j}=0$ vacuum.

If both $\ell$ and $r$ are odd, then the origin of moduli space is no longer a solution to the equations of motion:

$$
\begin{aligned}
\operatorname{det} M_{\ell r}= & \left(\Lambda_{1}^{b} \Lambda_{3}^{b} \ldots \Lambda_{\ell}^{b}\right)\left(\bar{\Lambda}_{1}^{b} \bar{\Lambda}_{3}^{b} \ldots \bar{\Lambda}_{r}^{b}\right)-\left(Z_{0} Z_{1} \Lambda_{3}^{b} \ldots \Lambda_{\ell}^{b}+Z_{0} \Lambda_{2}^{b} Z_{3} \ldots \Lambda_{\ell}^{b}+\ldots\right)\left(\bar{\Lambda}_{1}^{b} \ldots \bar{\Lambda}_{r}^{b}\right) \\
& -\left(\Lambda_{1}^{b} \ldots \Lambda_{\ell}^{b}\right)\left(\bar{Z}_{0} \bar{Z}_{1} \bar{\Lambda}_{3}^{b} \ldots \bar{\Lambda}_{r}^{b}+\bar{Z}_{0} \bar{\Lambda}_{2}^{b} \bar{Z}_{3} \ldots \bar{\Lambda}_{r}^{b}+\ldots\right)+\ldots
\end{aligned}
$$

To satisfy this constraint, either $\langle M\rangle \neq 0,\left\langle Z_{\text {even }} Z_{\text {odd }}\right\rangle \neq 0$, or $\left\langle\bar{Z}_{\text {even }} \bar{Z}_{\text {odd }}\right\rangle \neq 0$. Different family symmetries are broken in each case, leaving different sets of independent operators.

In the $\langle M\rangle \neq 0$ vacuum where $M_{j}^{i}$ is proportional to $\delta_{j}^{i}$, $\mathrm{SU}(4)_{L} \times \mathrm{SU}(4)_{R}$ is broken to its diagonal subgroup SU(4) ${ }_{d}$. The fields $Q_{\ell}$ and $\bar{Q}_{r}$ transform under $\mathrm{SU}(4)_{d}$ as $\bar{\square}$ and $\square$, respectively, while the meson $M$ decomposes as

$$
\bar{\square} \otimes \square=\mathbf{1} \oplus \mathbf{A d j}: \quad \mathbf{M}_{\ell \mathbf{r}} \longrightarrow\left(\operatorname{Tr} \mathbf{M}_{\ell \mathbf{r}}\right) \oplus\left(\mathbf{M}_{\ell \mathbf{r}}-\operatorname{Tr} \mathbf{M}_{\ell \mathbf{r}}\right) .
$$

In the $\mathrm{U}(1)_{A} \times \mathrm{U}(1)_{B}$ preserving vacuum with $Z_{i}=\bar{Z}_{j}=0$, it is possible to write $\bar{K}_{r}$ and $U_{1}$ either in terms of $K_{\ell}$ and $M_{\ell r}$, or $K_{\ell}$ and $U_{1}$ in terms of $\bar{K}_{r}$ and $M_{\ell r}$. Therefore, we can either remove the set $\left\{K_{\ell}, U_{1}, \operatorname{Tr} M\right\}$ or $\left\{\bar{K}_{r}, U_{1}, \operatorname{Tr} M\right\}$. This degeneracy is related to the fact that $K_{\ell}$ and $\bar{K}_{r}$ have the same transformation properties un$\operatorname{der} \mathrm{SU}(4)_{d} \times \mathrm{U}(1)_{A} \times \mathrm{U}(1)_{B} \times \mathrm{U}(1)_{R}$.

If instead $\langle M\rangle=0$ and $\left\langle Z_{\text {even }} Z_{\text {odd }}\right\rangle \neq 0$, only $\mathrm{U}(1)_{A}$ is broken in the vacuum. One " $\left(Z_{\text {even }}+Z_{\text {odd }}\right)$ " linear combination determined by the ratio of the expectation values 
becomes massive, and all sixteen $M_{j}^{i}$ degrees of freedom remain independent. The operator $\bar{K}_{r}$ is not redundant in this vacuum: the $Z_{L} \bar{K}_{r}$ equation of motion includes a term $Z_{\text {even }} Z_{\text {odd }} \bar{K}_{r}$ which is not small. The set of redundant operators is $\left\{K_{\ell}, U_{1},\left(Z_{\text {even }}+Z_{\text {odd }}\right)\right\}$.

Finally, if the nonzero expectation value is $\left\langle\bar{Z}_{\text {even }} \bar{Z}_{\text {odd }}\right\rangle$, then $\mathrm{U}(1)_{B}$ is broken. As we would expect from the left-right symmetry, the redundant operators are $\left\{\bar{K}_{r}, U_{1},\left(\bar{Z}_{\text {even }}+\right.\right.$ $\left.\left.\bar{Z}_{\text {odd }}\right)\right\}$ in this vacuum. It is also possible to break a linear combination of $\mathrm{U}(1)_{A}$ and $\mathrm{U}(1)_{B}$ if $\left\langle Z_{\text {even }} Z_{\text {odd }}\right\rangle \neq 0$ and $\left\langle\bar{Z}_{\text {even }} \bar{Z}_{\text {odd }}\right\rangle \neq 0$.

Anomaly matching. We have discussed six distinct cases with maximal symmetry in the vacuum, based on $\ell$ and $r$. Below, we show a summary of our results for each case:

\begin{tabular}{|c|c|l|}
\hline$(\ell, r)$ & Broken symmetry & Redundant operators \\
\hline (even, even) & None & None \\
(odd, even) & None & $\left\{\bar{K}_{r}, U_{1}, \bar{Z}_{\text {even }}\right\}$ \\
(even, odd) & None & $\left\{K_{\ell}, U_{1}, Z_{\text {even }}\right\}$ \\
\hline & $\mathrm{SU}(4)_{L} \times \mathrm{SU}(4)_{R}$ & $\left\{K_{\ell}\right.$ or $\left.\bar{K}_{r}, U_{1}, \operatorname{Tr} M_{\ell r}\right\}$ \\
(odd, odd) & $\mathrm{U}(1)_{A}$ & $\left\{K_{\ell}, U_{1},\left(Z_{\text {even }}+Z_{\text {odd }}\right)\right\}$ \\
& $\mathrm{U}(1)_{B}$ & $\left\{\bar{K}_{r}, U_{1},\left(\bar{Z}_{\text {even }}+\bar{Z}_{\text {odd }}\right)\right\}$ \\
\hline
\end{tabular}

For the remaining symmetries and operators in each case, we have verified that the anomaly coefficients match the UV theory. There are 21 matching conditions for each of the first three cases, 17 for the fourth case, and 12 each for the final two cases. Although some of these coefficients are related to each other via the left-right symmetry, the explicit calculation is lengthy and not very illuminating.

Let us also consider points on the moduli space with nonzero $\left\langle Z_{i}\right\rangle$ or $\left\langle\bar{Z}_{j}\right\rangle$, where none of the operators in the set $\mathcal{F}$ are redundant. In these vacua $\mathrm{U}(1)_{A} \times \mathrm{U}(1)_{B}$ is spontaneously broken, and the infrared operators should obey anomaly matching conditions for the remaining symmetries.

For the odd $\ell$, even $r$ case, $\mathrm{U}(1)_{A}$ is broken by $\left\langle Z_{i}\right\rangle \neq 0$ for some $Z_{i}$. After $\mathrm{U}(1)_{A}$ is broken, $\left\{U_{1}, \bar{Z}_{\text {even }}\right\}$ form an anomaly-neutral pair: their $\mathrm{U}(1)_{B, R}$ charges are opposite, so all of the $\mathrm{U}(1)^{3}$ and gravitational $\mathrm{U}(1)$ anomalies cancel. The fermionic part of $\bar{K}_{r}$ is neutral under $\mathrm{U}(1)_{B} \times \mathrm{U}(1)_{R}$, and it is in a real representation of $\mathrm{SU}(4)_{R}$ : therefore, $\bar{K}_{r}$ contributes nothing to the remaining anomaly coefficients. Thus, the t' Hooft anomaly matching conditions are also satisfied in the $\left\langle Z_{i}\right\rangle \neq 0$ vacuum where the operators $\left\{\bar{K}_{r}, U_{1}, \bar{Z}_{\text {even }}\right\}$ are independent degrees of freedom.

In the even- $\ell$, odd- $r$ models, the operators $\left\{K_{\ell}, U_{1}, Z_{\text {even }}\right\}$ are restored as independent degrees of freedom when $\left\langle\bar{Z}_{j}\right\rangle \neq 0$ and $\mathrm{U}(1)_{B}$ is spontaneously broken. Applying the left-right transformation to the above results, the introduction of $\left\{K_{\ell}, U_{1}, Z_{\text {even }}\right\}$ has no net effect on the anomaly coefficients once $\mathrm{U}(1)_{B}$ is removed. Finally, when $\left\langle Z_{i}\right\rangle \neq 0$ and $\left\langle\bar{Z}_{j}\right\rangle \neq 0$ in the odd- $\ell$, odd- $r$ models, the operators $\left\{K_{\ell}, U_{1}, Z_{\text {even }}\right\}$ are restored as independent degrees of freedom without contributing to the anomaly coefficients of the remaining symmetries. Both $\mathrm{U}(1)_{A}$ and $\mathrm{U}(1)_{B}$ are broken in this case. 


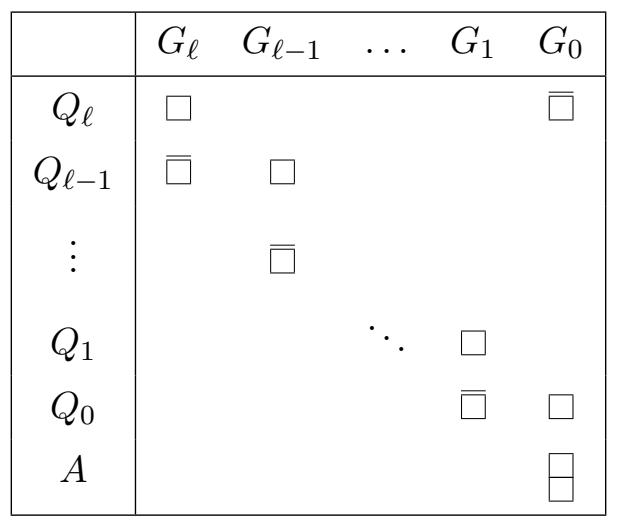

Table 9. Above, we show the matter fields of the SU(4) ring extension to the $A+4 Q+4 \bar{Q}$ model.

Flows. Our proposed s-confining extensions to the SU(4) model pass several consistency checks. As a final test, let us spontaneously break the gauge group by giving large expectation values to the gauge invariant operators, as in section 2.3. For example, $\left\langle M_{\ell r}\right\rangle \gg \Lambda$ breaks SU $(4)^{\ell+r+1}$ to $\mathrm{SU}(3)^{\ell+r+1}$, leaving $\boxminus+4 \square+3 \square$ matter charged under SU $(3)_{0}$. Three of the $\square$ fields come from the $G_{0} \times G_{1}$ bifundamental $Q_{0}$, while the fourth comes from

$$
\mathrm{SU}(4) \rightarrow \mathrm{SU}(3): \quad \boxminus \rightarrow \boxminus \oplus \square .
$$

Note that $\square=\bar{\square}$ for SU(3), so that there are effectively $(3+1)$ flavors of $(\square+\bar{\square})$ charged under $\mathrm{SU}(3)_{0}$. The low-energy theory is a left-right extension of $F=4, N=3$ SUSY QCD, where an $\mathrm{SU}(3)_{L} \times \mathrm{SU}(3)_{R}$ subgroup of the family $\mathrm{SU}(4)_{L} \times \mathrm{SU}(4)_{R}$ is gauged. In section 3.3 we consider such models in more detail.

Along flat directions with $\langle\operatorname{Pf} A\rangle \gg \Lambda_{0}, \mathrm{SU}(4)_{0}$ is broken to $\mathrm{Sp}(4)$, leaving an $(\ell, r)$ product group extension of the s-confining $\mathrm{Sp}(4):(4+4) \square$ model. In this theory an $\mathrm{SU}(4)_{L} \times \mathrm{SU}(4)_{R}$ subgroup of the $\mathrm{SU}(8)$ family symmetry is gauged. We discuss models of this type in section 3.2 .

Summary. In every $(\ell, r)$ model with $(\ell, r) \neq(0,0)$, there are quantum deformations to the classical moduli space. The origin remains on the moduli space unless both $\ell$ and $r$ are odd. In the mixed case where only one of $\{\ell, r\}$ is odd, eight of the fields become redundant in the vacua which conserve $\mathrm{U}(1)_{A} \times \mathrm{U}(1)_{B}$. If $\ell$ and $r$ are both even, all of the infrared operators in eq. (3.3) are independent, interacting degrees of freedom even at the origin of moduli space. Due to the existence of a dynamically generated superpotential and the possibility of confinement without chiral symmetry breaking, we conclude that the $(\ell, r)$ models are s-confining if $\ell$ and $r$ are not both odd.

SU(4) ring extension. Before moving on to consider other types of models, let us extend the $(\ell, r)$ model even further by gauging a diagonal subgroup $G_{d}$ of the family $\mathrm{SU}(4)_{L} \times \mathrm{SU}(4)_{R}$ symmetry. This connects the left and right ends of the $(\ell, r)$ extension as shown in table 9 , so that different models are labelled by the sum $(\ell+r)$. Models of this type appear in deconstructions of $5 \mathrm{~d}$ gauge theories, as in [8]. 
Although the baryon operators $\operatorname{Pf} A$ and $\operatorname{det} Q_{i}$ are unaffected by the ringlike nature of the product gauge group, there is now only one gauge-invariant meson operator: $\operatorname{Tr} M=$ $\operatorname{Tr}\left(Q_{0} Q_{1} \ldots Q_{\ell}\right)$. For any group $G_{i}$, the adjoint operator

$$
\left(\hat{M}_{i}\right)_{\beta}^{\alpha}=\left(Q_{i} Q_{i+1} \ldots Q_{\ell} Q_{0} \ldots Q_{i-1}\right)_{\beta}^{\alpha}-\frac{1}{4}(\operatorname{Tr} M) \delta_{\beta}^{\alpha}
$$

is a degree of freedom in the limit where $G_{i}$ is weakly gauged, and can be used to create gauge-invariant operators of the type $\operatorname{Tr}\left(\hat{M}_{i} \hat{M}_{i}\right)$ and $\operatorname{Tr}\left(\hat{M}_{i}^{3}\right)$. In this notation, $Q_{-1}=Q_{\ell}$ for the $i=0$ case.

Even when these operators have large expectation values, the gauge group is not completely broken. It has been shown [23] in the $\mathrm{SU}(N)^{k}$ extension to $F=N$ SUSY QCD that at an arbitrary point on the moduli space has a remaining $\mathrm{U}(1)^{3}$ gauge group. In the $A+4 Q+4 \bar{Q}$ model it is also possible to set $\langle\operatorname{Pf} A\rangle \gg \Lambda_{0}$, so that $\mathrm{SU}(4)_{0}$ is broken to $\operatorname{Sp}(4)$. This reduces the rank of the group by one, but is not sufficient to break $\mathrm{U}(1)^{3}$ completely. Therefore, the $\mathrm{SU}(4)$ ring extension has a Coulomb branch, and is not s-confining.

\section{$3.2 \operatorname{Sp}(2 m)$ with $(2 m+4)$ quarks}

In section 2.3, we found that the $\mathrm{SU}(N)^{k}$ extension of the $A+4 Q+N \bar{Q}$ model flows to an $\mathrm{Sp}(2 m) \times \mathrm{SU}(2 m)^{k-1}$ theory. In the limit where $\mathrm{Sp}(2 m)$ is much more strongly coupled than the $\mathrm{SU}(2 m)$ groups, the $(2 m+4)$ quarks confine to produce the operator $M=\left(Q^{2}\right)$, which transforms in the $\boxminus$ representation under the approximate $\mathrm{SU}(2 m+4)$ family symmetry.

The fields $Q$ and $M$ have the following charges:

\begin{tabular}{|c|c|cc|}
\hline & $\mathrm{Sp}(2 m)$ & $\mathrm{SU}(2 m+4)$ & $\mathrm{U}(1)_{R}$ \\
\hline$Q$ & $\square$ & $\square$ & $1 /(m+2)$ \\
\hline$M$ & & $\square$ & $2 /(m+2)$ \\
\hline
\end{tabular}

A dynamically generated superpotential

$$
W_{d}=\frac{\operatorname{Pf} M}{\Lambda^{2 m+1}}
$$

reproduces the classical constraints on the $Q_{i}$ fields.

In the product gauge group model shown in table 10, an $\mathrm{SU}(2 m)$ subgroup of the family symmetry is gauged and new bifundamental fields are added to cancel the anomalies. The family $\mathrm{SU}(2 m+4)$ is explicitly broken to $\mathrm{SU}(2 m) \times \mathrm{SU}(4) \times \mathrm{U}(1)$, under which the meson $M$ decomposes as

$$
\boxminus \longrightarrow(\boxminus, \mathbf{1} ;-4) \oplus(\square, \square ; m-2) \oplus(\mathbf{1}, \emptyset ; 2 m): \quad M \longrightarrow M_{A} \oplus M_{Q} \oplus M_{0},
$$

and the dynamically generated superpotential becomes

$$
W_{d} \longrightarrow \frac{M_{A}^{m-1} M_{Q}^{2} M_{0}}{\Lambda^{2 m+1}} .
$$

Including the bifundamental field $\bar{Q}_{1}$, the $\mathrm{SU}(2 m)_{1}$ charged matter in the confined phase of $\operatorname{Sp}(2 m)$ is $M_{A}+4 M_{Q}+2 m \bar{Q}_{1}$, which is expected to s-confine. 


\begin{tabular}{|c|c|c|ccc|c|}
\hline & $\mathrm{SU}(4)_{L}$ & $\mathrm{Sp}(2 m)$ & $\mathrm{SU}(2 m)_{1}$ & $\ldots$ & $\mathrm{SU}(2 m)_{k}$ & $\mathrm{SU}(2 m)_{R}$ \\
\hline$Q_{L}$ & $\square$ & $\square$ & & & & \\
$\bar{Q}_{0}$ & & $\square$ & $\square$ & & & \\
$\bar{Q}_{1}$ & & & $\square$ & & & \\
$\vdots$ & & & & $\ddots$ & & \\
$\bar{Q}_{k-1}$ & & & & & $\square$ & \\
$\bar{Q}_{k}$ & & & & & $\square$ & $\square$ \\
\hline$\left(Q_{L}^{2}\right)$ & $\square$ & & & & & \\
$\left(Q_{L} \bar{Q}_{0}\right)$ & $\square$ & & $\square$ & & & \\
$\left(\bar{Q}_{0}^{2}\right)$ & & & $\square$ & & & \\
$\bar{Q}_{1}$ & & & $\square$ & & & \\
$\vdots$ & & & $\square$ & & \\
$\bar{Q}_{k}$ & & & & $\ddots$ & $\square$ & $\square$ \\
\hline
\end{tabular}

Table 10. An $\operatorname{Sp}(2 m) \times \mathrm{SU}(2 m)^{k}$ model is shown, which is expected to s-confine. At the bottom of the table, we list the degrees of freedom in the confined phase of $\mathrm{Sp}(2 m)$. Subsequent confinement follows the pattern of the $A+4 Q+N \bar{Q}$ model.

This model can also be derived using the deconfinement technique of Berkooz [1], by treating the matter field $A$ as a bound state of two quarks transforming in the fundamental representation of a new $\operatorname{Sp}(N)$.

\subsection{SUSY QCD}

A product group extension to $F=N+1$ SUSY QCD can be derived from the $N=3$ case of $A+4 Q+N \bar{Q}$. In $\mathrm{SU}(3)$, the $\boxminus$ representation is the same as $\bar{\square}$, so that the $G_{1}$ matter is effectively $4 \square+4 \bar{\square}$. By gauging the $\mathrm{SU}(3)$ family symmetry of the $\bar{Q}$ and adding a sequence of bifundamental fields $\bar{Q}_{i}$, we have found a product group extension to SUSY QCD.

For larger values of $N$, let us gauge an $\mathrm{SU}(N)$ subgroup of the $\mathrm{SU}(N+1)_{R}$ family symmetry as shown in table 11. After $\mathrm{SU}(N)_{1}$ confinement, the hadrons are $(Q \bar{q}),\left(Q \bar{Q}_{1}\right)$, $\left(Q^{N}\right),\left(\bar{Q}_{1}^{N}\right)$, and $\left(\bar{q} \bar{Q}_{1}^{N-1}\right)$, which transform under $\mathrm{SU}(N)_{2}$ and the family symmetries as described in table 12. Under $\mathrm{SU}(N)_{2}$ there are $(N+1)(\square+\square)$ matter fields, which is consistent with the index constraint for s-confinement.

For this theory to be s-confining, it must be shown that the dynamically generated superpotential from $\mathrm{SU}(N)_{1}$ does not prevent the operators $\left(Q \bar{Q}_{1}\right)$ and $\left(\bar{q} \bar{Q}_{1}^{N-1}\right)$ from varying independently; that the infrared operators obey the appropriate anomaly matching conditions; and that the origin is on the moduli space. The additional gauge groups are likely to introduce quantum-modified constraints between some of the operators, which may induce chiral symmetry breaking in some cases. 


\begin{tabular}{|r|c|cc|c|}
\hline & $\mathrm{SU}(N+1)_{L}$ & $\mathrm{SU}(N)_{1}$ & $\mathrm{SU}(N)_{2}$ & $\mathrm{SU}(N)_{R}$ \\
\hline$Q$ & $\square$ & $\square$ & & \\
$\bar{q}$ & & $\overline{1}$ & & \\
$\bar{Q}_{1}$ & & $\overline{1}$ & $\square$ & \\
$\bar{Q}_{2}$ & & & $\bar{\square}$ & $\square$ \\
\hline
\end{tabular}

Table 11. A single product group extension to the s-confining SUSY QCD theory is shown.

\begin{tabular}{|r|c|c|c|}
\hline & $\mathrm{SU}(N+1)_{L}$ & $\mathrm{SU}(N)_{2}$ & $\mathrm{SU}(N)_{R}$ \\
\hline$(Q \bar{q})$ & $\square$ & & \\
$\left(Q^{N}\right)$ & $\square$ & & \\
$\left(\bar{Q}_{1}^{N}\right)$ & & & \\
\hline$\left(Q \bar{Q}_{1}\right)$ & $\square$ & $\square$ & \\
$\left(\bar{q} \bar{Q}_{1}^{N-1}\right)$ & & $\square$ & \\
$\bar{Q}_{2}$ & & $\square$ & $\square$ \\
\hline
\end{tabular}

Table 12. The operators shown above describe the degrees of freedom after $\mathrm{SU}(N)_{1}$ confines.

This theory can also be extended by gauging an $\mathrm{SU}(N)$ subgroup of $\mathrm{SU}(N+1)_{L}$, so that the most general product group extension is $\mathrm{SU}(N)^{\ell} \times \mathrm{SU}(N)_{0} \times \mathrm{SU}(N)^{r}$. Based on the behavior of the $(\ell, r) A+4 Q+4 \bar{Q}$ model for odd $\ell$ and $r$, we expect that some of the $(\ell, r)$ SUSY QCD models also break chiral symmetry.

Alternating gauge groups. The $F=N+1$ model can also be extended by gauging the entire $\mathrm{SU}(N+1)$ family symmetry. In this case, the gauge group has the alternating form $\mathrm{SU}(N) \times \mathrm{SU}(N+1) \times \mathrm{SU}(N) \times \mathrm{SU}(N+1) \times \ldots$, with the series of bifundamental fields shown in table 13. The matter content is simpler in this case, as all of the fields are $\mathrm{SU}(N+1) \times \mathrm{SU}(N)$ bifundamentals. When $\mathrm{SU}(N)_{1}$ confines, we are left with the operators shown in table 14. Under $\mathrm{SU}(N+1)_{2}$, there are $(N+1)$ flavors of $\square+\bar{\square}$ which is expected to confine with chiral symmetry breaking. Many of the $G_{2}$ singlets we would naïvely construct, such as $\left(Q \bar{Q}_{1}\right)\left(\bar{Q}_{1}^{N}\right)$, are set to zero by the equations of motion, so $G_{2}$ confinement leaves the following charged fields:

\begin{tabular}{|r|c|c|c|}
\hline & $\mathrm{SU}(N+1)_{L}$ & $\mathrm{SU}(N)_{3}$ & $\mathrm{SU}(N+1)_{R}$ \\
\hline$\left(Q^{N}\right)$ & $\square$ & & \\
$\left(Q \bar{Q}_{1} \bar{Q}_{2}\right)$ & $\square$ & $\square$ & \\
$\bar{Q}_{3}$ & & $\square$ & $\square$ \\
\hline
\end{tabular}

After $G_{1} \times G_{2}$ confinement, the low energy theory is simply $F=N+1$ sUSY QCD with some gauge singlet fields. 


\begin{tabular}{|c|c|ccc|c|}
\hline & $\mathrm{SU}(N+1)_{L}$ & $\mathrm{SU}(N)_{1}$ & $\mathrm{SU}(N+1)_{2}$ & $\mathrm{SU}(N)_{3}$ & $\mathrm{SU}(N+1)_{R}$ \\
\hline$Q$ & $\square$ & $\square$ & & & \\
$\bar{Q}_{1}$ & & $\square$ & $\square$ & & \\
$\bar{Q}_{2}$ & & & $\square$ & $\square$ & \\
$\bar{Q}_{3}$ & & & & $\square$ & $\square$ \\
\hline
\end{tabular}

Table 13. With alternating $\mathrm{SU}(N)$ and $\mathrm{SU}(N+1)$ gauge groups, the s-confining SUSY QCD theory can be extended by adding a string of bifundamentals.

\begin{tabular}{|r|c|cc|c|}
\hline & $\mathrm{SU}(N+1)_{L}$ & $\mathrm{SU}(N+1)_{2}$ & $\mathrm{SU}(N)_{3}$ & $\mathrm{SU}(N+1)_{R}$ \\
\hline$\left(Q^{N}\right)$ & $\square$ & & & \\
$\left(Q \bar{Q}_{1}\right)$ & $\square$ & $\square$ & & \\
$\left(\bar{Q}_{1}^{N}\right)$ & & $\overline{ }$ & & \\
$\bar{Q}_{2}$ & & $\bar{\square}$ & $\square$ & \\
$\bar{Q}_{3}$ & & & $\bar{\square}$ & $\square$ \\
\hline
\end{tabular}

Table 14. The operators in the confined phase of SU $(N)_{1}$ have the same form as SUSY QCD with $N=F$, but with a dynamically generated superpotential.

Both product group models based on SUSY QCD have the potential to be s-confining, and may be promising directions for future study.

\subsection{Other models}

Of the s-confining theories listed in [5], there are only a few models possessing non-Abelian family symmetries larger than the gauge group. We have already discussed the $\mathrm{SU}(N)$ models with $A+4 Q+N \bar{Q}$ and $(N+1)(Q+\bar{Q})$, as well as the $\operatorname{Sp}(2 m)$ model with $(2 m+4) Q$. There are two remaining cases based on $\operatorname{Sp}(2 m)$ with $A+6 Q[24,25]$. If $m=2$ or $m=3$, an $\mathrm{SU}(4)$ or $\mathrm{SU}(6)$ subgroup of the family symmetry can be gauged. In this section, we show that the product group extensions do not exhibit s-confinement.

Sp(6) with $\boldsymbol{A}+\mathbf{6 Q}$. Consider the $m=3$ case with just one extra product group. In table 15, we show the matter fields above and below the $\operatorname{Sp}(6)$ confinement scale. In the confined phase of $\mathrm{Sp}(6)$, the $\mathrm{SU}(6)$ index sum becomes

$$
\sum_{j} \mu_{j}-\mu_{G}=3 \cdot(6-2)+6 \cdot 1-2 \cdot 6=+6
$$

so the product group does not s-confine. It may be possible to remove some of the degrees of freedom by adding a nonzero tree-level superpotential, but this is outside the scope of the current study. 


\begin{tabular}{|r|cc|c|}
\hline & $\mathrm{Sp}(6)$ & $\mathrm{SU}(6)$ & $\mathrm{SU}(6)_{R}$ \\
\hline$A$ & $\boxminus$ & & \\
$Q$ & $\square$ & $\square$ & \\
$\bar{Q}$ & & $\square$ & $\square$ \\
\hline$\left(A^{2}\right)$ & & & \\
$\left(A^{3}\right)$ & & & \\
$\left(Q^{2}\right)$ & & $\square$ & \\
$(Q A Q)$ & & $\square$ & \\
$\left(Q A^{2} Q\right)$ & & $\square$ & \\
$\bar{Q}$ & \multicolumn{3}{|c|}{} \\
\hline
\end{tabular}

Table 15. An s-confining $\mathrm{Sp}(6)$ theory is extended by gauging the $\mathrm{SU}(6)$ global symmetry of the quarks. The resulting theory is not s-confining.

\begin{tabular}{|r|c|cc|c|}
\hline & $\mathrm{SU}(2)_{L}$ & $\mathrm{Sp}(4)$ & $\mathrm{SU}(4)$ & $\mathrm{SU}(6)_{R}$ \\
\hline$Q_{L}$ & $\square$ & $\square$ & & \\
$A$ & & $\square$ & & \\
$Q_{R}$ & & $\square$ & $\square$ & \\
$\bar{Q}$ & & & $\square$ & $\square$ \\
\hline
\end{tabular}

Table 16. For the s-confining $\mathrm{Sp}(4)$ theory, an $\mathrm{SU}(4)$ subgroup of the $\mathrm{SU}(6)$ flavor symmetry is gauged.

$\operatorname{Sp}(4)$ with $\boldsymbol{A}+\mathbf{6 Q}$. In the $\mathrm{Sp}(4)$ case shown in table 16, an $\mathrm{SU}(4)$ subgroup of the $\mathrm{SU}(6)$ family symmetry is gauged. The set of $\mathrm{Sp}(4)$ invariants is

$$
\mathcal{F}=\left\{\left(A^{2}\right) ;\left(Q_{L}^{2}\right),\left(Q_{L} Q_{R}\right),\left(Q_{R}^{2}\right) ;\left(Q_{L} A Q_{L}\right),\left(Q_{L} A Q_{R}\right),\left(Q_{R} A Q_{R}\right)\right\} .
$$

The operators $\left(Q_{L} Q_{R}\right)$ and $\left(Q_{L} A Q_{R}\right)$ are bifundamentals of $\mathrm{SU}(2) \times \mathrm{SU}(4)$, while $\left(Q_{R}^{2}\right)$ and $\left(Q_{R} A Q_{R}\right)$ transform as $(\mathbf{1}, \boxminus)$. The other hadrons are gauge singlets. Together with $\bar{Q}$, the $\mathrm{SU}(4)$ charged matter is $2 \square+4 \square+4 \bar{\square}$, with the index sum

$$
\sum_{j} \mu_{j}-\mu_{G}=2(2)+4(1)+4(1)-2 \cdot 4=+4 .
$$

Therefore, the $\mathrm{Sp}(4)$ product group extension to $\mathrm{Sp}(4):(A+6 Q)$ is also not s-confining.

\section{Conclusion}

For several s-confining theories, we find product gauge group models with the following properties: 
- All infrared degrees of freedom are gauge invariant composite fields;

- The infrared physics is described by a smooth effective theory, which is valid everywhere on the moduli space (including the origin);

- There is a dynamically generated superpotential.

This allows confinement without symmetry breaking, even when the quantum and classical moduli spaces are different. In particular, this behavior may be found in the following models:

$$
\mathrm{SU}(N): A+4 Q+N \bar{Q} \quad \operatorname{Sp}(2 m):(2 m+4) Q \quad \mathrm{SU}(N):(N+1)(Q+\bar{Q}) .
$$

In this paper we argue that the $A+4 Q+N \bar{Q}$ and $\mathrm{Sp}(2 m):(2 m+4) Q$ product group models s-confine. Based on less rigorous arguments we suggest two product group extensions of SUSY QCD which may also be s-confining, but a more detailed analysis is required. It is also entirely possible that there are many other s-confining product group theories unrelated to the models considered in this paper.

In the $A+4 Q+N \bar{Q}$ model with $N=4$, we consider a set of product group extensions of the form $G_{L}^{\ell} \times G_{0} \times G_{R}^{r}$. When $\ell$ and $r$ are both odd, the chiral symmetry is necessarily broken in the vacuum, so the theory is not s-confining. If instead the sum $(\ell+r)$ is odd, then the origin remains on the quantum-deformed moduli space, and some of the infrared operators become redundant in the symmetry-enhanced vacua. Finally, if $\ell$ and $r$ are both even, we find that all of the operators are interacting degrees of freedom in the neighborhood of the origin. In each case, there is a dynamically generated superpotential.

One feature of the product group models is the lack of small gauge-invariant operators, which has a promising phenomenological application to composite axion models. After lifting some of the flat directions, a Peccei-Quinn U(1) symmetry may be dynamically broken when the gauge group confines, producing a light composite axion. If the product gauge group is suitably large, the Peccei-Quinn symmetry is protected against the explicit symmetry breaking effects which would otherwise be induced by higher-dimensional operators. We explore this option in a recent paper [26].

Another promising direction for future study is to treat the product gauge groups as $k$ site decompositions of $5 \mathrm{~d}$ SUSY theories. Exact calculations in $\mathcal{N}=2$ SUSY may provide us with a better understanding of the $4 \mathrm{~d} \mathcal{N}=1$ models considered in this paper.

\section{Acknowledgments}

We would like to thank Csaba Csaki, Yuri Shirman, Tim M.P. Tait, Flip Tanedo, and Arvind Rajaraman for helpful conversations. This work was supported in part by NSF Grants PHY-1316792 and PHY-1620638, and in part by Simons Investigator Award \#376204. 


\section{A Derivation of classical constraints}

In this section we find the classical constraints between gauge singlet operators in the $A+4 Q+N \bar{Q}$ model, along with the coefficients in the dynamically generated superpotential. It is useful to consider a particular non-trivial solution of the $D$ flatness conditions.

\section{A.1 D-flat directions}

The auxiliary gluon scalar fields have interactions from the Kähler potential given by $V=\frac{1}{2} D^{a} D^{a}$, where

$$
D^{a}=-g\left(Q_{i}^{\star \alpha}\left(T_{\square}^{a}\right)_{\alpha}^{\beta} Q_{\beta}^{i}+\bar{Q}_{\alpha}^{\star j}\left(T_{\bar{\square}}^{a}\right)_{\beta}^{\alpha} \bar{Q}_{j}^{\alpha}+A^{\star \beta \alpha}\left(T_{\square}^{a}\right)_{\alpha \beta}^{\delta \epsilon} A_{\delta \epsilon}\right) .
$$

Ground state solutions are given by $D^{a} D^{a}=0$. Equation (A.1) can be simplified by replacing $T_{\bar{\square}}$ and $T_{\square}$ with $T_{\square}^{a}$ :

$$
\left(T_{\square}^{a}\right)_{\beta}^{\alpha}=-\left(T_{\square}^{a}\right)_{\alpha}^{\beta}, \quad\left(T_{\square}^{a}\right)_{\alpha \beta}^{\delta \epsilon}=\left(T_{\square}^{a}\right)_{\alpha}^{\delta} \delta_{\beta}^{\epsilon}+\delta_{\alpha}^{\delta}\left(T_{\square}^{a}\right)_{\beta}^{\epsilon} .
$$

With this substitution, we may write $D^{a}$ as

$$
D^{a}=-g\left(Q_{i}^{\star \alpha} Q_{\beta}^{i}-\bar{Q}_{j}^{\alpha} \bar{Q}_{\beta}^{\star j}+2 A^{\star \alpha \gamma} A_{\gamma \beta}\right)\left(T_{\square}^{a}\right)_{\alpha}^{\beta} .
$$

The indices $i$ and $j$ refer to $\mathrm{SU}(4)_{L}$ and $\mathrm{SU}(N)_{R}$, respectively, while $\alpha, \beta$ and $\gamma$ correspond to the gauge group. The generators $T_{\square}^{a}$ span the set of traceless $N \times N$ matrices, so if the fields satisfy

$$
Q_{i}^{\star \alpha} Q_{\beta}^{i}-\bar{Q}_{j}^{\alpha} \bar{Q}_{\beta}^{\star j}+2 A^{\star \alpha \gamma} A_{\gamma \beta}=\rho \delta_{\beta}^{\alpha}
$$

for any constant $\rho$, then $D^{a}=0$. It is useful to define the matrices $d, \bar{d}$, and $d_{A}$ as follows:

$$
d_{\beta}^{\alpha}=Q_{i}^{\star \alpha} Q_{\beta}^{i}, \quad \bar{d}_{\beta}^{\alpha}=\bar{Q}_{j}^{\alpha} \bar{Q}_{\beta}^{\star j}, \quad\left(d_{A}\right)_{\beta}^{\alpha}=A^{\star \alpha \gamma} A_{\gamma \beta},
$$

so that eq. (A.4) can be written as

$$
d_{\beta}^{\alpha}-\bar{d}_{\beta}^{\alpha}+2\left(d_{A}\right)_{\beta}^{\alpha}=\rho \delta_{\beta}^{\alpha} .
$$

Each $d$ term defined above is invariant under the $\mathrm{SU}(4)_{L} \times \mathrm{SU}(N)_{R}$ flavor transformations.

By rotating the $\mathrm{SU}(N)$ color basis, it is possible to block-diagonalize the matrix $A$ such that the only non-zero entries are $A_{12}=-A_{21}=\sigma_{1}, A_{34}=-A_{43}=\sigma_{2}$, etc. For even $\mathrm{SU}(N=2 m)$, this continues until $\sigma_{m}=A_{N-1, N}$. In this basis, the $d_{A}$ matrix is diagonal and equal to

$$
\left(d_{A}\right)_{\beta}^{\alpha}=\operatorname{Diag}\left(\left|\sigma_{1}\right|^{2},\left|\sigma_{1}\right|^{2},\left|\sigma_{2}\right|^{2},\left|\sigma_{2}\right|^{2}, \ldots,\left|\sigma_{m}\right|^{2},\left|\sigma_{m}\right|^{2}\right),
$$

with Pf $A=\sigma_{1} \sigma_{2} \ldots \sigma_{m}$. For odd $N=2 m+1, \sigma_{m}=A_{N-2, N-1}$, and $A_{j N}=0$ for all $j=1 \ldots N$. The $d_{A}$ matrix is again diagonal, but with $\left(d_{A}\right)_{N}^{N}=0$.

$$
\left(d_{A}\right)_{\beta}^{\alpha}=\operatorname{Diag}\left(\left|\sigma_{1}\right|^{2},\left|\sigma_{1}\right|^{2},\left|\sigma_{2}\right|^{2},\left|\sigma_{2}\right|^{2}, \ldots,\left|\sigma_{m}\right|^{2},\left|\sigma_{m}\right|^{2}, 0\right) .
$$

The Pfaffian, $\operatorname{Pf} A$, is not defined for odd-dimensional matrices.

It is not generally possible to simultaneously diagonalize $d_{A}, d$, and $\bar{d}$. This is a departure from SUSY QCD: in this case, if $\bar{d}$ is diagonal, then $d_{\beta}^{\alpha}=\bar{d}_{\beta}^{\alpha}+\rho \delta_{\beta}^{\alpha}$ must also be diagonal. Once $d_{A}$ is added, this condition is relaxed. 


\section{A.2 Special cases}

In this section we consider the $\langle\phi\rangle \gg \Lambda$ limit along particular flat directions in which $d_{A}$, $d$, and $\bar{d}$ happen to be diagonal. Let us begin with the $N=2 m$ case:

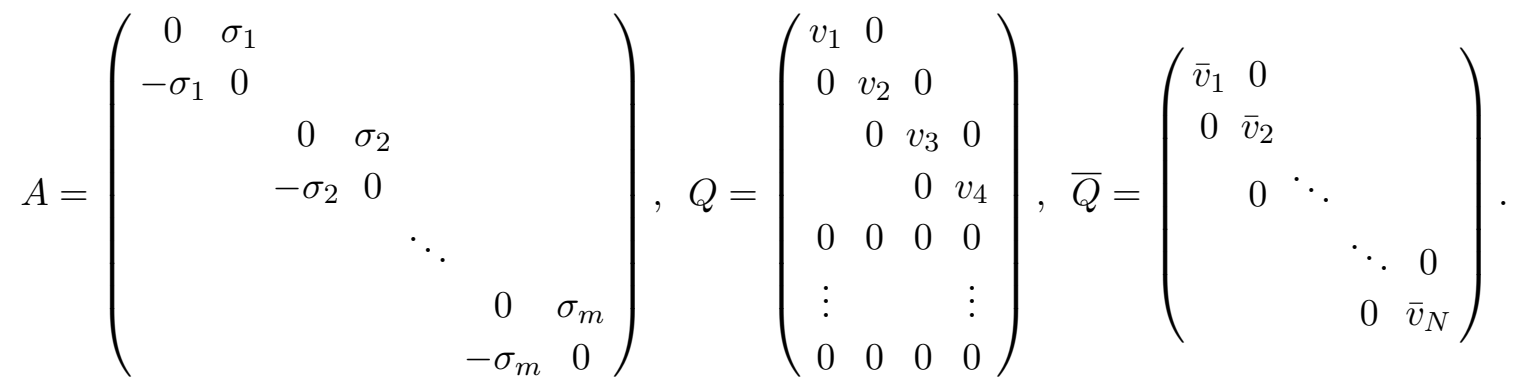

In this vacuum, the matrices $d_{A}, d$ and $\bar{d}$ are:

$$
\begin{aligned}
d_{A} & =\operatorname{Diag}\left(\left|\sigma_{1}\right|^{2},\left|\sigma_{1}\right|^{2},\left|\sigma_{2}\right|^{2},\left|\sigma_{2}\right|^{2}, \ldots,\left|\sigma_{m}\right|^{2},\left|\sigma_{m}\right|^{2}\right) \\
d & =\operatorname{Diag}\left(\left|v_{1}\right|^{2},\left|v_{2}\right|^{2},\left|v_{3}\right|^{2},\left|v_{4}\right|^{2}, 0, \ldots, 0\right) \\
\bar{d} & =\operatorname{Diag}\left(\left|\bar{v}_{1}\right|^{2},\left|\bar{v}_{2}\right|^{2},\left|\bar{v}_{3}\right|^{2}, \ldots,\left|\bar{v}_{N-1}\right|^{2},\left|\bar{v}_{N}\right|^{2}\right)
\end{aligned}
$$

subject to the constraint

$$
d_{\alpha}^{\alpha}-\bar{d}_{\alpha}^{\alpha}+2\left(d_{A}\right)_{\alpha}^{\alpha}=\rho .
$$

In the classical limit, the gauge-invariant operators are

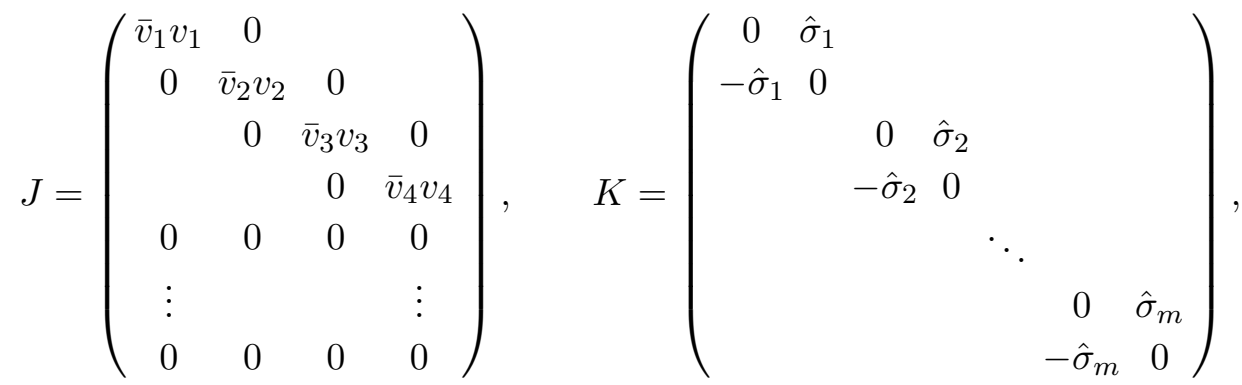

$$
\begin{aligned}
& V=\left(\begin{array}{cccc}
0 & V_{12} & 0 & 0 \\
-V_{12} & 0 & 0 & 0 \\
0 & 0 & 0 & V_{34} \\
0 & 0 & -V_{34} & 0
\end{array}\right), \quad \begin{aligned}
U & =\sigma_{1} \sigma_{2} \ldots \sigma_{m} \\
\mathcal{W} & =v_{1} v_{2} v_{3} v_{4} \sigma_{3} \ldots \sigma_{m} \\
Z & =\bar{v}_{1} \bar{v}_{2} \bar{v}_{3} \ldots \bar{v}_{N},
\end{aligned}
\end{aligned}
$$

where we define

$$
V_{12} \equiv\left(v_{1} v_{2}\right) \sigma_{2} \sigma_{3} \ldots \sigma_{m}, \quad V_{34} \equiv \sigma_{1}\left(v_{3} v_{4}\right) \sigma_{3} \ldots \sigma_{m}, \quad \hat{\sigma}_{i} \equiv \sigma_{i} \bar{v}_{2 i-1} \bar{v}_{2 i}
$$

for $i=1 \ldots m$.

In the $N=2 m+1$ case we add a row and column to $A$, with $A_{\alpha, N}=A_{N, \beta}=0$ for all $\alpha$ and $\beta$. The form of $\bar{Q}$ is left unchanged, but we add a nontrivial $N^{\text {th }}$ row to $Q_{N}^{i}$ with 
entries $q^{i} \neq 0$. With these modifications, the matrices $d_{A}, d$ and $\bar{d}$ become

$$
\begin{aligned}
d_{A} & =\operatorname{Diag}\left(\left|\sigma_{1}\right|^{2},\left|\sigma_{1}\right|^{2},\left|\sigma_{2}\right|^{2},\left|\sigma_{2}\right|^{2}, \ldots,\left|\sigma_{m}\right|^{2},\left|\sigma_{m}\right|^{2}, 0\right) \\
d & =\operatorname{Diag}\left(\left|v_{1}\right|^{2},\left|v_{2}\right|^{2},\left|v_{3}\right|^{2},\left|v_{4}\right|^{2}, 0, \ldots, 0, \sum_{i}\left|q_{i}\right|^{2}\right) \\
\bar{d} & =\operatorname{Diag}\left(\left|\bar{v}_{1}\right|^{2},\left|\bar{v}_{2}\right|^{2},\left|\bar{v}_{3}\right|^{2}, \ldots,\left|\bar{v}_{N-1}\right|^{2},\left|\bar{v}_{N}\right|^{2}\right)
\end{aligned}
$$

and the gauge-invariant operators are

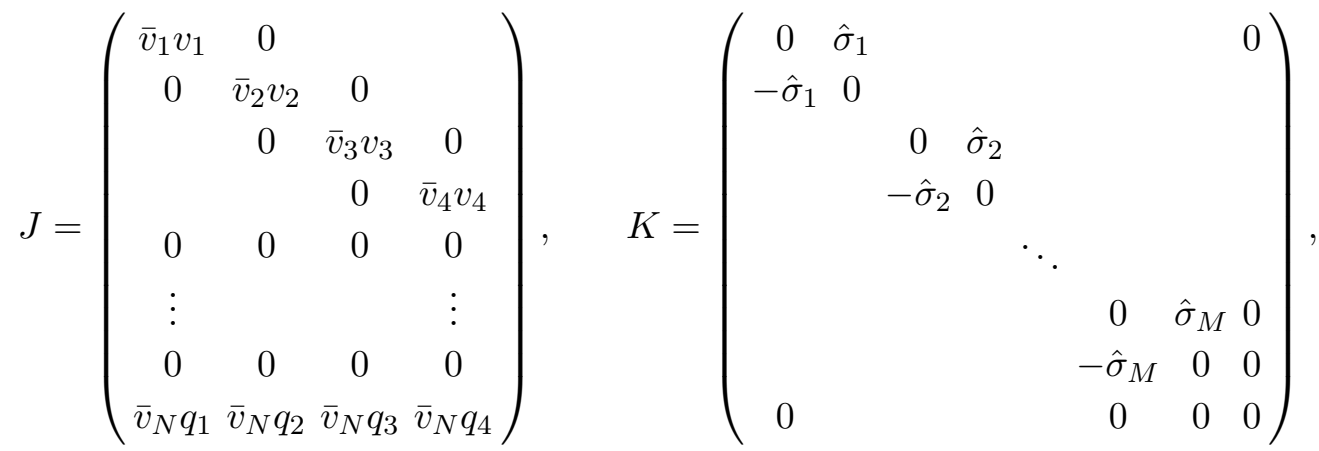

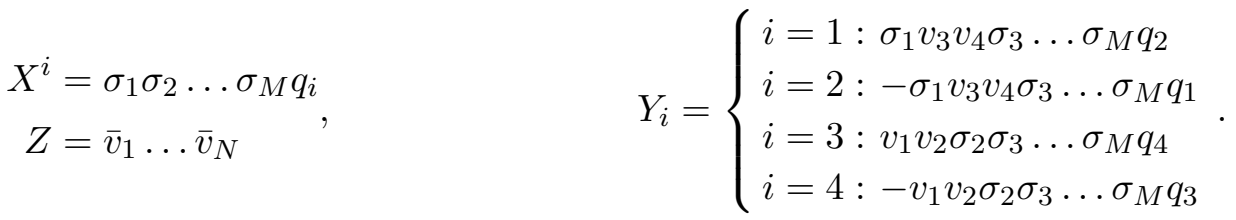

Classical constraints. The dynamically generated superpotential has the form $W \sim$ $A^{N-2} Q^{4} \bar{Q}^{N}$. For odd $N$, there are three ways to contract the gauge indices:

$$
\begin{aligned}
W_{d}= & \frac{\alpha}{\Lambda^{b}}\left(X^{i} Y_{i} Z+\beta_{1} \epsilon_{i_{1} \ldots i_{4}} \epsilon^{j_{1} \ldots j_{N}} X^{i_{1}}\left(K_{j_{1} j_{2}} \ldots K_{j_{N-4} j_{N-3}}\right) J_{j_{N-2}}^{i_{2}} J_{j_{N-1}}^{i_{3}} J_{j_{N}}^{i_{4}}\right. \\
& \left.+\beta_{2} \epsilon^{j_{1} \ldots j_{N}} Y_{i}\left(K_{j_{1} j_{2}} \ldots K_{j_{N-2} j_{N-1}}\right) J_{j_{N}}^{i}\right),
\end{aligned}
$$

while for even $N$ there are five terms:

$$
\begin{aligned}
W_{d}= & \frac{\alpha}{\Lambda^{b}}\left(U \mathcal{W} Z+\gamma_{1} \epsilon_{i_{1} \ldots i_{4}} V^{i_{1} i_{2}} V^{i_{3} i_{4}} Z+\gamma_{2} \epsilon_{j_{1} \ldots j_{N}} \epsilon_{i_{1} \ldots i_{4}} U\left(K_{j_{1} j_{2}} \ldots K_{j_{N-5}, j_{N-4}}\right)\left(J_{j_{N-3}}^{i_{1}} \ldots J_{j_{N}}^{i_{4}}\right)\right. \\
& \left.+\gamma_{3} \epsilon_{j_{1} \ldots j_{N}} \epsilon_{i_{1} \ldots i_{4}} V^{i_{1} i_{2}}\left(K_{j_{1} j_{2} \ldots K_{j_{N-3}, j_{N-2}}}\right)\left(J_{j_{N-1}}^{i_{3}} J_{j_{N}}^{i_{4}}\right)+\gamma_{4} \mathcal{W} \operatorname{Pf} K\right) .
\end{aligned}
$$

The relationships between the coefficients are determined by matching the equations of motion from $W_{d}$ to the classical constraints on the operators.

In the classical limit for even $N$, it follows from eq. (A.15) that

$$
\operatorname{Pf} V=V_{12} V_{34}=\left(\sigma_{1} \sigma_{2} v_{1} v_{2} v_{3} v_{4}\right)\left(\sigma_{3} \ldots \sigma_{m}\right)^{2}=U \cdot Z,
$$

for example, so that

$$
\gamma_{1}=-\frac{1}{2^{2} 2 !}
$$


Applying this technique to other products of gauge invariant operators, we find

$$
\gamma_{2}=-\frac{1}{2^{m-2}(m-2) ! 4 !}, \quad \gamma_{3}=+\frac{1}{4 \cdot 2^{m-1}(m-1) !}, \quad \gamma_{4}=-1 .
$$

For odd $N$ the relevant classical constraints have the form

$$
\begin{aligned}
X^{i} Z & =-\beta_{2} \epsilon^{j_{1} \ldots j_{N}}\left(K_{j_{1} j_{2}} \ldots K_{j_{N-2} j_{N-1}}\right) J_{j_{N}}^{i} \\
Y_{i} Z & =-\beta_{1} \epsilon_{i i_{2} i_{3} i_{4}} \epsilon^{j_{1} \ldots j_{N}}\left(K_{j_{1} j_{2}} \ldots K_{j_{N-4} j_{N-3}}\right) J_{j_{N-2}}^{i_{2}} J_{j_{N-1}}^{i_{3}} J_{j_{N}}^{i_{4}} .
\end{aligned}
$$

Based on eqs. (A.20) and (A.21),

$$
\begin{aligned}
X^{i} Z & =\left(\sigma_{1} \ldots \sigma_{m} q_{i}\right)\left(\bar{v}_{1} \ldots \bar{v}_{N}\right) \\
Y_{i} Z & =\left(\sigma_{1} v_{3} v_{4} \sigma_{3} \ldots \sigma_{m} q_{2}\right)\left(\bar{v}_{1} \ldots \bar{v}_{N}\right),
\end{aligned}
$$

which when matched to the corresponding products of $J$ and $K$ imply that

$$
\beta_{1}=-\frac{1}{2^{m-1}(m-1) ! 3 !}, \quad \beta_{2}=-\frac{1}{2^{m} m !} .
$$

In both cases the overall constant $\alpha$ has no effect on the equations of motion, and cannot be calculated from the classical constraints.

Open Access. This article is distributed under the terms of the Creative Commons Attribution License (CC-BY 4.0), which permits any use, distribution and reproduction in any medium, provided the original author(s) and source are credited.

\section{References}

[1] M. Berkooz, The Dual of supersymmetric $\mathrm{SU}(2 K)$ with an antisymmetric tensor and composite dualities, Nucl. Phys. B 452 (1995) 513 [hep-th/9505067] [INSPIRE].

[2] P. Pouliot, Duality in SUSY SU(N) with an antisymmetric tensor, Phys. Lett. B 367 (1996) 151 [hep-th/9510148] [INSPIRE].

[3] E. Poppitz, Y. Shadmi and S.P. Trivedi, Duality and exact results in product group theories, Nucl. Phys. B 480 (1996) 125 [hep-th/9605113] [INSPIRE].

[4] C. Csáki, M. Schmaltz and W. Skiba, A Systematic approach to confinement in $N=1$ supersymmetric gauge theories, Phys. Rev. Lett. 78 (1997) 799 [hep-th/9610139] [INSPIRE].

[5] C. Csáki, M. Schmaltz and W. Skiba, Confinement in $N=1$ SUSY gauge theories and model building tools, Phys. Rev. D 55 (1997) 7840 [hep-th/9612207] [INSPIRE].

[6] T. Hirayama and K. Yoshioka, Duality between simple group gauge theories and some applications, Phys. Rev. D 59 (1999) 105005 [hep-th/9811119] [INSPIRE].

[7] F. Brünner and V.P. Spiridonov, A duality web of linear quivers, Phys. Lett. B 761 (2016) 261 [arXiv: 1605.06991] [INSPIRE].

[8] C. Csáki, J. Erlich, C. Grojean and G.D. Kribs, 4D constructions of supersymmetric extra dimensions and gaugino mediation, Phys. Rev. D 65 (2001) 015003 [hep-ph/0106044] [INSPIRE]. 
[9] C. Csáki, J. Erlich, V.V. Khoze, E. Poppitz, Y. Shadmi and Y. Shirman, Exact results in $5 D$ from instantons and deconstruction, Phys. Rev. D 65 (2002) 085033 [hep-th/0110188] [INSPIRE].

[10] W. Skiba and D. Tucker-Smith, Localized fermions and anomaly inflow via deconstruction, Phys. Rev. D 65 (2002) 095002 [hep-ph/0201056] [INSPIRE].

[11] S. Chang and H. Georgi, Quantum modified mooses, Nucl. Phys. B 672 (2003) 101 [hep-th/0209038] [INSPIRE].

[12] N. Seiberg, Electric-magnetic duality in supersymmetric non-Abelian gauge theories, Nucl. Phys. B 435 (1995) 129 [hep-th/9411149] [InSPIRE].

[13] K.A. Intriligator and N. Seiberg, Phases of $N=1$ supersymmetric gauge theories in four-dimensions, Nucl. Phys. B 431 (1994) 551 [hep-th/9408155] [INSPIRE].

[14] N. Seiberg, The Power of duality: Exact results in 4D SUSY field theory, Int. J. Mod. Phys. A 16 (2001) 4365 [hep-th/9506077] [INSPIRE].

[15] A.C. Davis, M. Dine and N. Seiberg, The Massless Limit of Supersymmetric QCD, Phys. Lett. B 125 (1983) 487 [INSPIRE].

[16] I. Affleck, M. Dine and N. Seiberg, Dynamical Supersymmetry Breaking in Four-Dimensions and Its Phenomenological Implications, Nucl. Phys. B 256 (1985) 557 [InSPIRE].

[17] N. Seiberg, Exact results on the space of vacua of four-dimensional SUSY gauge theories, Phys. Rev. D 49 (1994) 6857 [hep-th/9402044] [INSPIRE].

[18] K.A. Intriligator and N. Seiberg, Lectures on supersymmetric gauge theories and electric-magnetic duality, Nucl. Phys. Proc. Suppl. B 45 (1996) 1 [hep-th/9509066] [INSPIRE].

[19] E. Poppitz and S.P. Trivedi, Some examples of chiral moduli spaces and dynamical supersymmetry breaking, Phys. Lett. B 365 (1996) 125 [hep-th/9507169] [INSPIRE].

[20] J. Terning, Duals for $\mathrm{SU}(N)$ SUSY gauge theories with an antisymmetric tensor: Five easy flavors, Phys. Lett. B 422 (1998) 149 [hep-th/9712167] [INSPIRE].

[21] K.A. Intriligator, "Integrating in" and exact superpotentials in 4d, Phys. Lett. B 336 (1994) 409 [hep-th/9407106] [INSPIRE].

[22] K.A. Intriligator and P. Pouliot, Exact superpotentials, quantum vacua and duality in supersymmetric $S P\left(N_{c}\right)$ gauge theories, Phys. Lett. B 353 (1995) 471 [hep-th/9505006] [INSPIRE].

[23] C. Csáki, J. Erlich, D.Z. Freedman and W. Skiba, $N=1$ supersymmetric product group theories in the Coulomb phase, Phys. Rev. D 56 (1997) 5209 [hep-th/9704067] [INSPIRE].

[24] P.L. Cho and P. Kraus, Symplectic SUSY gauge theories with antisymmetric matter, Phys. Rev. D 54 (1996) 7640 [hep-th/9607200] [INSPIRE].

[25] C. Csáki, W. Skiba and M. Schmaltz, Exact results and duality for $\operatorname{Sp}(2 N)$ SUSY gauge theories with an antisymmetric tensor, Nucl. Phys. B 487 (1997) 128 [hep-th/9607210] [INSPIRE].

[26] B. Lillard and T.M.P. Tait, A Composite Axion from a Supersymmetric Product Group, arXiv: 1707.04261 [INSPIRE]. 